\title{
A Systematic Review of Metabolite-to-Drug Ratios of Pharmaceuticals in Hair for Forensic Investigations
}

\author{
Karen Rygaard *(D), Kristian Linnet $(D)$ and Sys Stybe Johansen $(1 D$ \\ Section of Forensic Chemistry, Department of Forensic Medicine, Faculty of Health and Medical Sciences, \\ University of Copenhagen, Frederik V's Vej 11, 2100 Copenhagen, Denmark; kristian.linnet@sund.ku.dk (K.L.); \\ sys.johansen@sund.ku.dk (S.S.J.) \\ * Correspondence: Karen.rygaard@sund.ku.dk
}

check for updates

Citation: Rygaard, K.; Linnet, K.; Johansen, S.S. A Systematic Review of Metabolite-to-Drug Ratios of Pharmaceuticals in Hair for Forensic Investigations. Metabolites 2021, 11, 686. https://doi.org/10.3390/ metabo11100686

Academic Editor: Cornelius Hess

Received: 27 August 2021

Accepted: 4 October 2021

Published: 6 October 2021

Publisher's Note: MDPI stays neutral with regard to jurisdictional claims in published maps and institutional affiliations.

Copyright: (c) 2021 by the authors. Licensee MDPI, Basel, Switzerland. This article is an open access article distributed under the terms and conditions of the Creative Commons Attribution (CC BY) license (https:// creativecommons.org/licenses/by/ $4.0 /)$.

\begin{abstract}
After ingestion, consumed drugs and their metabolites are incorporated into hair, which has a long detection window, ranging up to months. Therefore, in addition to conventional blood and urine analyses, hair analysis can provide useful information on long-term drug exposure. Meta-boliteto-drug (MD) ratios are helpful in interpreting hair results, as they provide useful information on drug metabolism and can be used to distinguish drug use from external contamination, which is otherwise a limitation in hair analysis. Despite this, the MD ratios of a wide range of pharmaceuticals have scarcely been explored. This review aims to provide an overview of MD ratios in hair in a range of pharmaceuticals of interest to forensic toxicology, such as antipsychotic drugs, antidepressant drugs, benzodiazepines, common opiates/opioids, etc. The factors influencing the ratio were evaluated. MD ratios of 41 pharmaceuticals were reported from almost 100 studies. MD ratios below 1 were frequently reported, indicating higher concentrations of the parent pharmaceutical than of its metabolite in hair, but wide-ranging MD ratios of the majority of pharmaceuticals were found. Intra- and interindividual differences and compound properties were variables possibly contributing to this. This overview presents guidance for future comparison and evaluation of MD ratios of pharmaceuticals.
\end{abstract}

Keywords: forensic science; hair analysis; head hair; pharmaceuticals; metabolite ratio

\section{Introduction}

Hair analysis can provide information regarding long-term drug exposure in addition to the conventional analyses of blood and urine analysis. Hair is a stable matrix with a long detection window, ranging up to months, depending on the length of the hair strands [1] Therefore, drug concentrations in hair contribute to information about previous drug consumption, which is of interest in the field of forensic toxicology.

After administration, drugs are absorbed, distributed and metabolized in the body, depending on the administration route. During metabolism, the drugs are chemically degraded by enzymes, and the presence of metabolites can be used as evidence of active intake $[2,3]$. The drugs and their metabolites are incorporated into the hair. Various mechanisms for drugs entering hair have been proposed, but the precise mechanisms involved remain unclear. Proposed mechanisms include incorporation of drugs by passive diffusion from blood capillaries into the growing hair cells, incorporation from deep skin compartments during hair shaft formation, or deposition by diffusion from sweat or sebum secretions into the completed hair shaft [3]. Hair from the posterior vertex of the scalp grows $1 \mathrm{~cm}$ per month on average, and by dividing hair samples collected from that area into specified segments, drug consumption for months can be tracked. Nevertheless, hair analysis results must be interpreted with caution because variables such as age, drug metabolism, external contamination, hair melanin content, cosmetic hair treatment, and interindividual variability may influence them [4]. Differentiation between external contamination and incorporation of drugs through ingestion is a general issue in hair analysis and is of particular concern when interpreting the results [5]. It has been 
proposed that the detection of relevant metabolites and calculation of metabolite-to-drug (MD) ratios can minimize misinterpretation [6,7].

MD ratios in blood and urine are commonly reported in the literature and have been used to evaluate short-term abstinence in postmortem cases as well as acute intoxication, as these are dependent on sampling and dosing time [8]. In contrast, MD ratios in hair represent the cumulative amount of drugs and metabolites, as these are deposited in hair from blood and sweat during metabolism. Therefore, MD ratios in hair provide useful information on drug metabolism and can be used to distinguish drug use from external contamination, and are thus a helpful tool to interpret hair results [9]. External contamination is a major problem, especially when evaluating drugs of abuse in hair, but contamination with pharmaceuticals can also be a problem when differentiating a single intake in terms of intoxication from a long-term intake.

MD ratios of drugs of abuse in hair have frequently been reported [10-13], but the literature is still very limited regarding pharmaceuticals in hair. Despite this, a wide range of pharmaceuticals is commonly encountered in postmortem and forensic toxicology cases $[14,15]$. The limited information on such drugs, their metabolites, and the related MD ratio makes it difficult to interpret and compare hair analysis results and to eventually evaluate drug intake. Therefore, a comprehensive overview of the MD ratios of pharmaceuticals in hair is needed.

The objective of this systematic review is to give an overview of MD ratios in hair for pharmaceuticals of interest in forensic toxicology such as antipsychotic drugs, antidepressant drugs, benzodiazepines, common opiates/opioids, etc. In addition, factors influencing the MD ratio will be evaluated.

\section{Methods}

\subsection{Information Sources and Search Strategy}

This systematic review was performed according to the Preferred Reporting Items for Systematic Reviews and Meta-Analyses (PRISMA) guidelines [16,17]. The review was not registered and a review protocol was not prepared. Relevant studies were identified by searching the PubMed (National Library of Medicine 1966 to present) and Embase (1974 to present) electronic databases. The last search was performed on March 182021. The searches were refined using the following MeSH terms and text words: drug OR pharmaceutical AND hair AND concentration OR determination AND metabolite OR "degradation product" AND analysis. No filters were used for the special study design. Additionally, the reference list of each included study was checked in order to identify further studies missed during the initial search.

\subsection{Exclusion Criteria}

Title and abstracts were screened based on the following exclusion criteria:

- Studies not written in English;

- Reviews, letters, book chapters, or conference abstracts;

- Studies not of interest in forensic toxicology, e.g., cancer-related studies;

- Studies not concerning the analysis of authentic human head hair;

- $\quad$ Studies on hair from children $\leq 3$ years old or unreported age;

- Studies not concerning antipsychotics, antidepressants, benzodiazepines, opioids / opiates (Except for morphine and codeine. See in Section 2.3);

- Studies not concerning other pharmaceuticals of interest in forensic toxicology, such as ketamine and methylphenidate;

- $\quad$ Studies on illegal drugs (New Psychoactive Substances (NPS)) or non-marketed drugs were excluded;

- Studies on compounds that cannot or have not been quantified in authentic hair (e.g., ethanol).

If even one of these criteria applied, the study was excluded from this review. 


\subsection{Study Selection}

A total of 94 studies were included in this review. The study selection process is shown in a flow chart (Figure 1) and prepared according to the PRISMA guidelines. Studies with reported MD ratios or studies in which the calculation of MD ratios were possible were included. The MD ratios of drugs of abuse that are not legal pharmaceuticals and their metabolites were excluded from the present review as they have previously been explored in detail [10-13]. In addition, non-marketed pharmaceuticals were excluded. The MD ratios of morphine and codeine were omitted from the present review because it was difficult to distinguish therapeutic use from abuse and thus differentiate the ratios after morphine, codeine, and heroin intake.

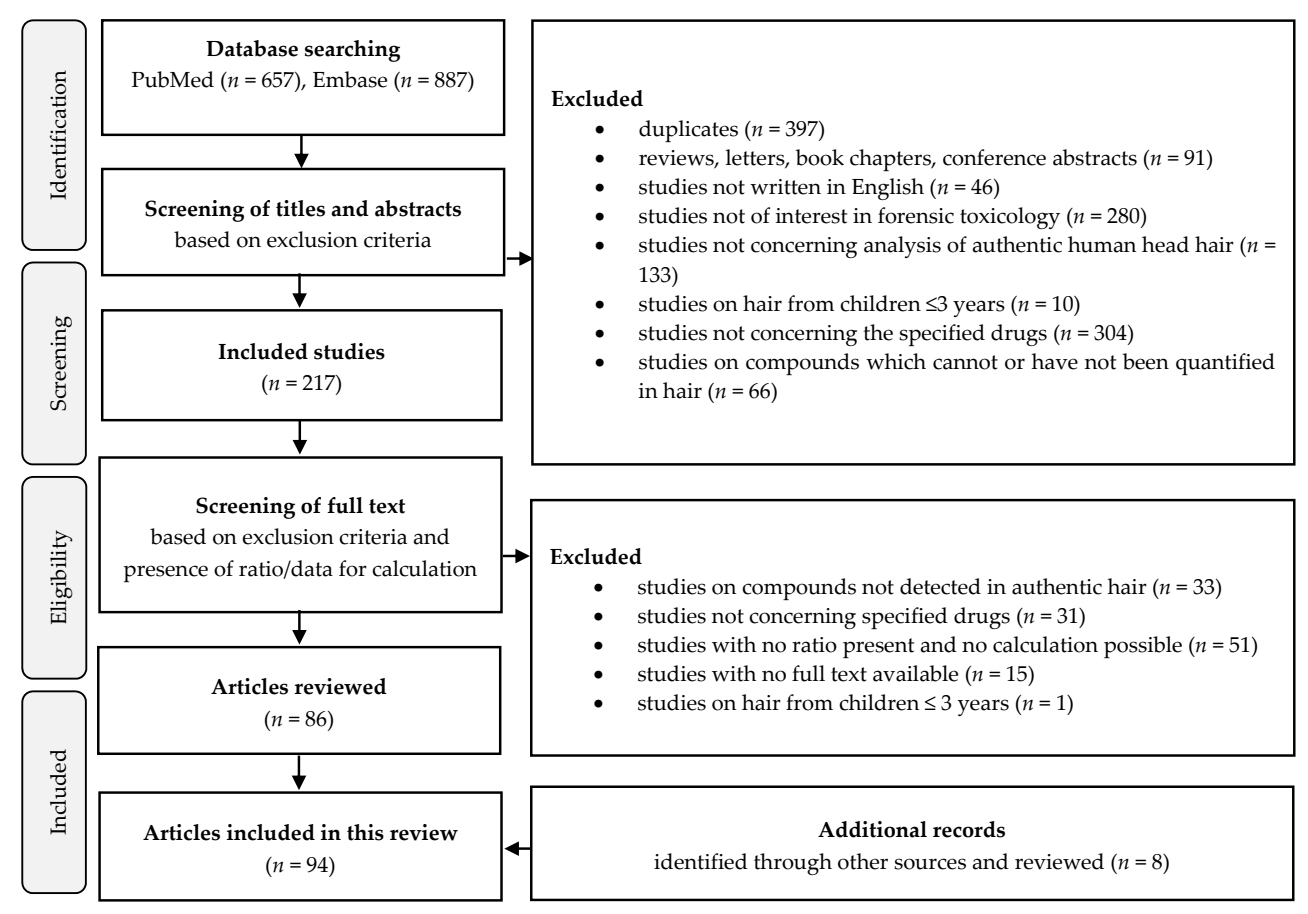

Figure 1. Flow chart of the study selection process developed according to PRISMA guidelines.

\subsection{Data Collection}

Information on MD ratios of each pharmaceutical was obtained directly from the studies, from the calculation of the reciprocal value of reported drug-to-metabolite ratios, or calculated from visible and unmerged drug and metabolite concentrations in studies where no ratio was reported. Only concentrations above the lower limit of quantification or below the upper limit of quantification were included. The calculated MD ratio range was reported in this paper, and for studies with $>10$ included individuals or segments, percentiles were also reported. In addition, the mean and median MD ratios were calculated, if possible. Studies with children aged $\geq 4$ years were included and the term children was used for individuals aged 4-9 years. MD ratios of pharmaceuticals and metabolites, which both can be ingested as the main drug, were excluded if intake of both was specified. This applied to diazepam and temazepam, among others.

\section{Results and Discussion}

MD ratios are helpful in interpreting hair results, as they provide useful information on drug metabolism and can be used to distinguish drug use from external contamination [7]. Despite this, the literature is still very limited regarding pharmaceuticals in hair. In order to obtain a sufficient amount of data, MD ratios reported in the literature together with MD ratios calculated from reported concentrations were included in this review. MD ratios for 41 pharmaceuticals from 94 studies have been included and summarized in Table 1. The table 
contains information on the MD ratios of the pharmaceuticals reported in each study, including the range and/or 10-90 percentiles of the MD ratios together with the median and mean MD ratios of each pharmaceutical, based on the information available. In addition, the associated case description, number of included individuals, information on the segmental analysis together with the total number of analyzed segments, and instrumental method for each study are included in the table. For pharmaceuticals with MD ratios reported by more than one study, the values were summarized with a combined ratio range representing the lowest and highest reported ratios, based on ranges and percentiles, as well as the reported median ratios presented as a range from the lowest reported median to the highest reported median. Percentiles of MD ratios were calculated for studies with more than 10 subjects/segments. For studies with only percentiles reported, this was used as the range of ratio. The MD ratios were summarized in this manner due to the inconstancy in the published MD ratios.

\subsection{Ratios in Hair}

Table 1 shows that MD ratios were most frequently reported for benzodiazepines and opioids/opiates, whereas reports on MD ratios of antidepressant and antipsychotic drugs were more limited. MD ratios of diazepam, ketamine, and methadone were reported from more than 14 studies each, while MD ratios of each antidepressant and antipsychotic pharmaceutical were reported from a maximum of seven studies.

Overall, MD ratios have been reported in the range from $<0.005$ to 110 based on all 41 pharmaceuticals, and for many of the included pharmaceuticals, wide-ranging ratios were seen both within and between studies. MD ratios of frequent pharmaceuticals from Table 1 are visualized in Figure 2, representing the summarized (combined) ratios.

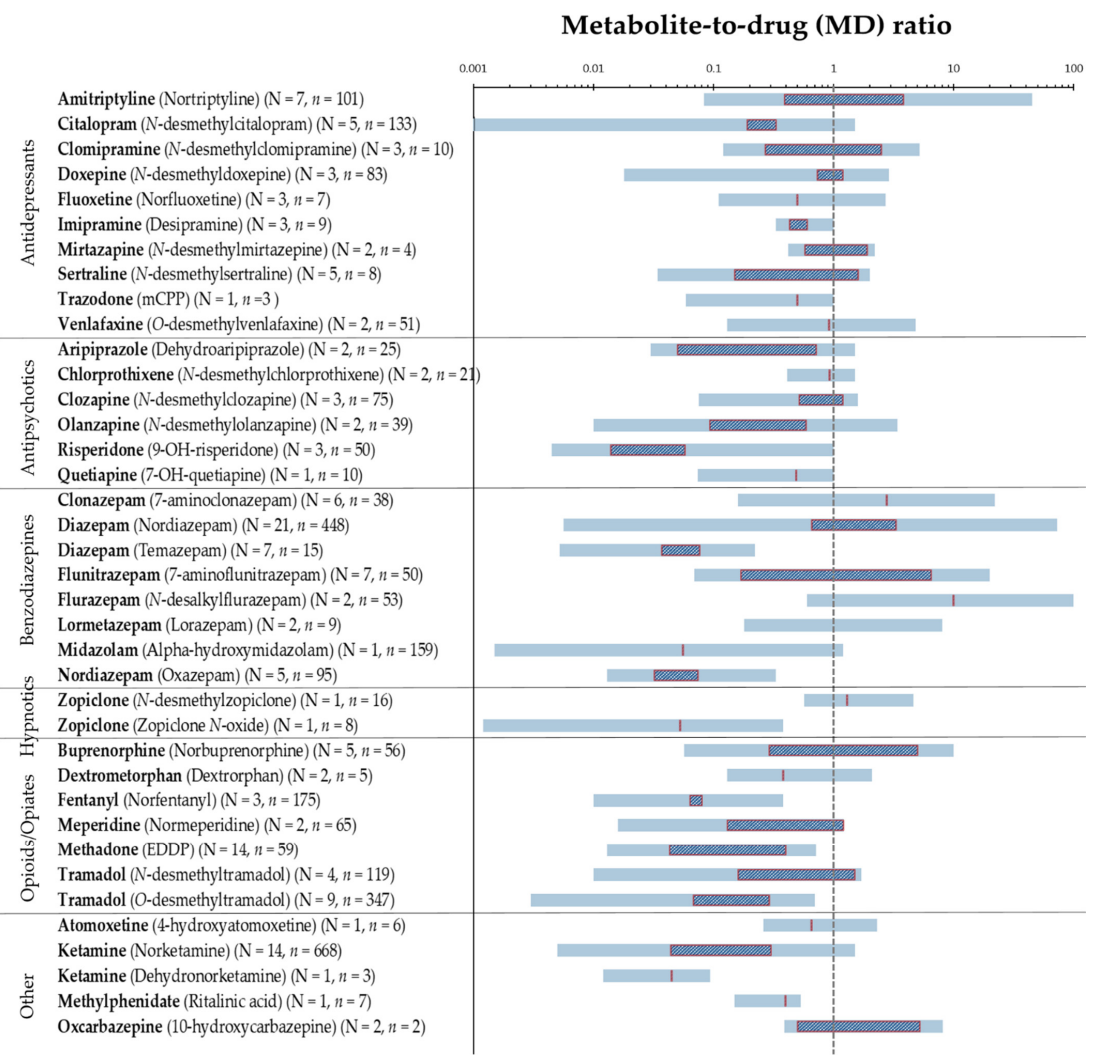

Figure 2. Visualization of MD ratios of 34 frequently reported pharmaceuticals, with N indicating the number of studies and $\mathrm{n}$ the number of individuals. Blue horizontal lines represent the MD ratio range from the lowest reported value to the highest. Red outlined squares represent range from the lowest reported median to highest, red vertical lines are cases with only one median value reported, and no line implies no reported median values. A logarithmic scale is used on the x-axis. The vertical dashed grey line indicates a MD ratio of 1.0. Cases with $\mathrm{N}=1$ and $n=1$ were excluded. 


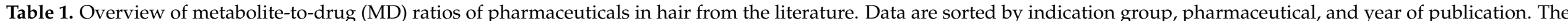

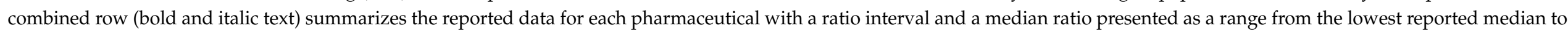
the highest reported median, if it appears in more than one study. Gray shades indicate self-calculated ratios from published concentrations.

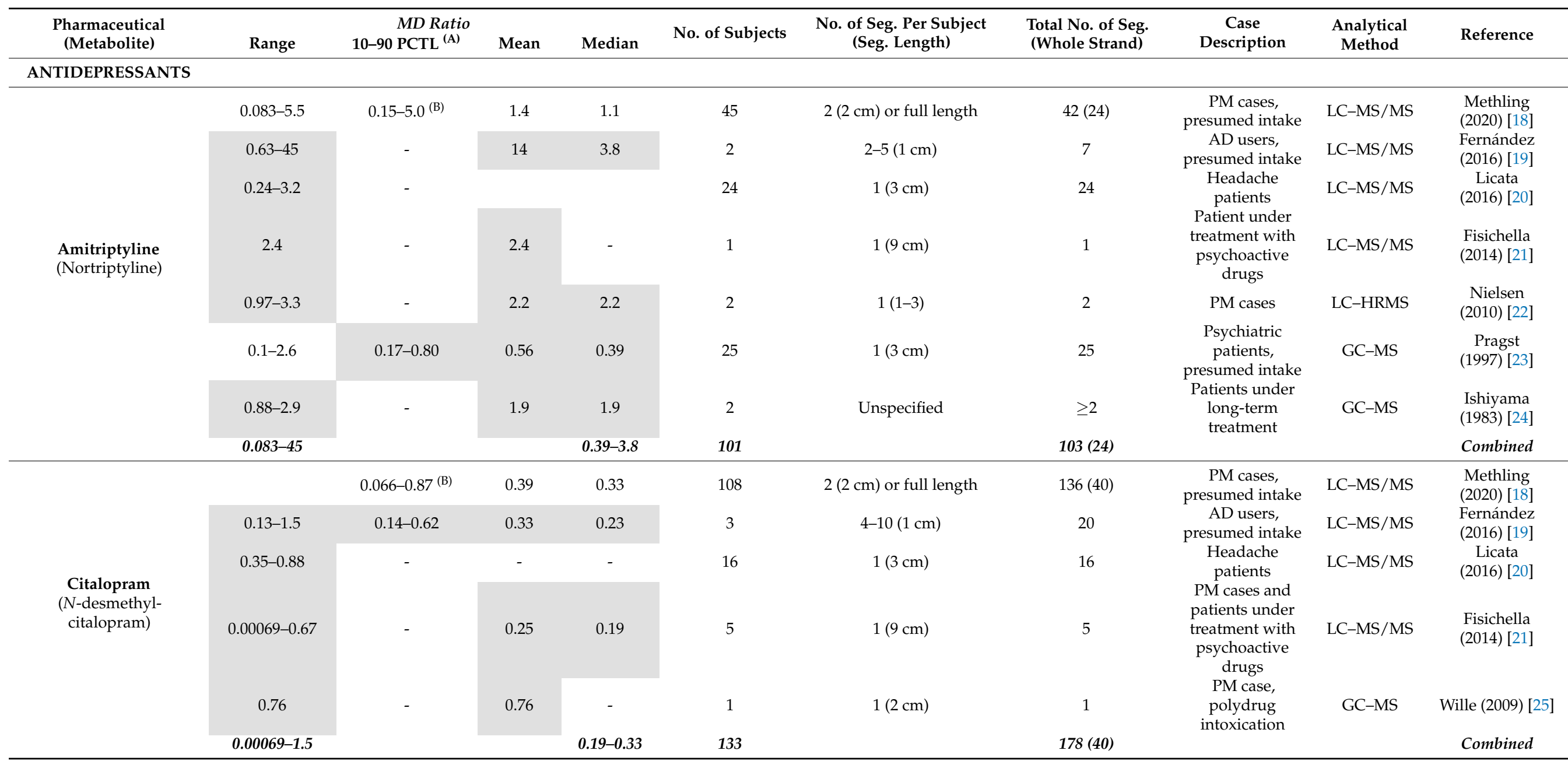


Table 1. Cont.

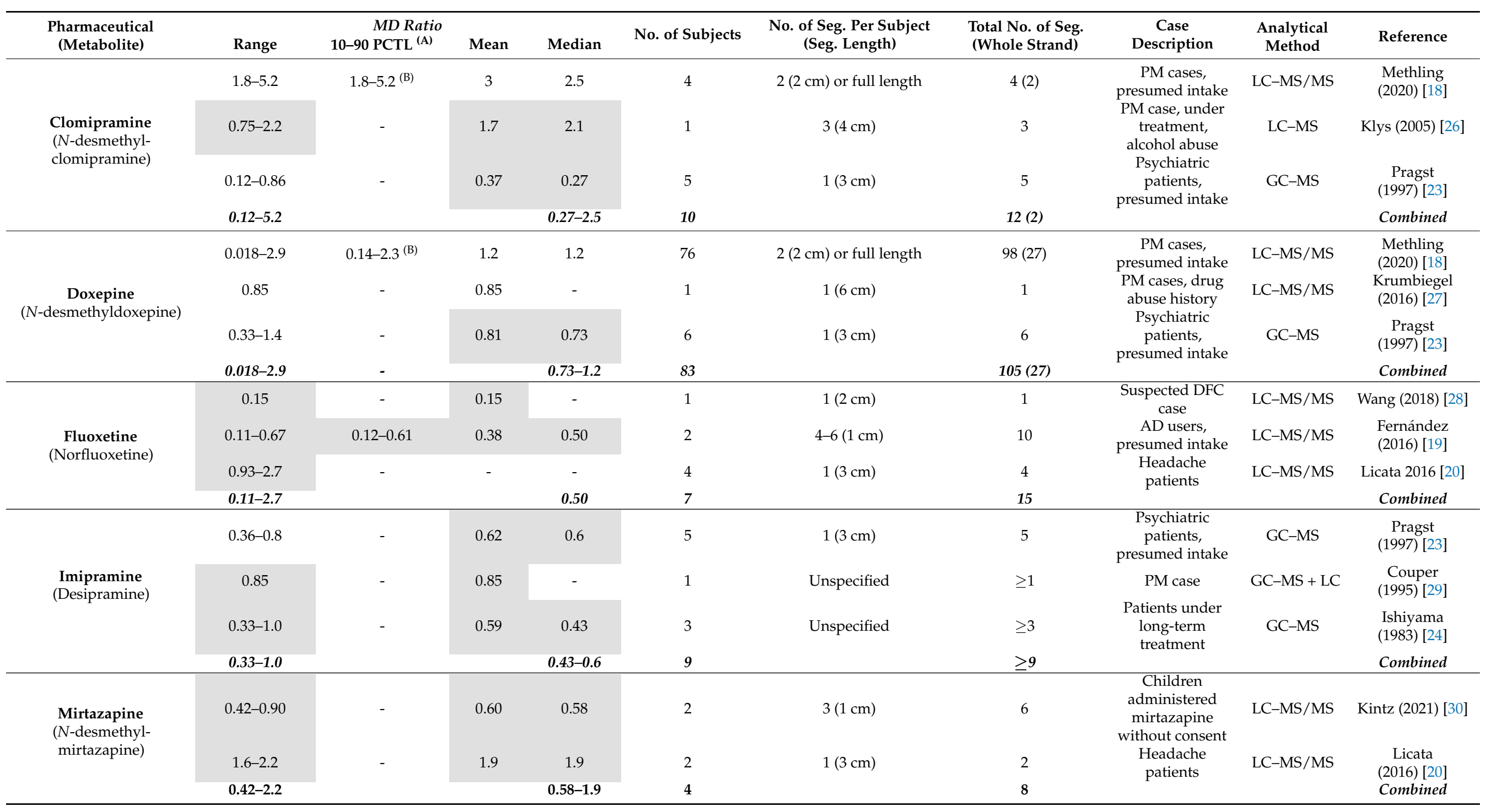


Table 1. Cont

\begin{tabular}{|c|c|c|c|c|c|c|c|c|c|c|}
\hline $\begin{array}{l}\text { Pharmaceutical } \\
\text { (Metabolite) }\end{array}$ & Range & $\begin{array}{l}\text { MD Ratio } \\
\text { 10-90 } \text { PCTL }^{\text {(A) }}\end{array}$ & Mean & Median & No. of Subjects & $\begin{array}{l}\text { No. of Seg. Per Subject } \\
\text { (Seg. Length) }\end{array}$ & $\begin{array}{l}\text { Total No. of Seg. } \\
\text { (Whole Strand) }\end{array}$ & $\begin{array}{c}\text { Case } \\
\text { Description }\end{array}$ & $\begin{array}{l}\text { Analytical } \\
\text { Method }\end{array}$ & Reference \\
\hline \multirow{6}{*}{$\begin{array}{c}\text { Sertraline } \\
\text { (N-desmethylsertraline) }\end{array}$} & 1.7 & - & 1.7 & - & 1 & $1(2 \mathrm{~cm})$ & 1 & \multirow{6}{*}{$\begin{array}{c}\text { Schizophrenic } \\
\text { patients under } \\
\text { treatment, in } \\
\text { compliance } \\
\text { Child suspected } \\
\text { accidental } \\
\text { intoxication } \\
\text { Headache } \\
\text { patients } \\
\text { AD and } \\
\text { anxiolytic drug } \\
\text { consumption } \\
\text { during } \\
\text { pregnancy } \\
\text { PM case, } \\
\text { polydrug } \\
\text { intoxication }\end{array}$} & LC-MS/MS & Wang (2019) [31] \\
\hline & $0.18-0.89$ & - & 0.50 & 0.50 & 1 & $6(2 \mathrm{~cm})$ & 6 & & LC-MS/MS & $\begin{array}{l}\text { Marchei } \\
\text { (2016) [32] }\end{array}$ \\
\hline & $0.79-2.0$ & - & - & - & 4 & $1(3 \mathrm{~cm})$ & 4 & & LC-MS/MS & $\begin{array}{c}\text { Licata } \\
(2016)[20]\end{array}$ \\
\hline & $0.034-0.16$ & - & 0.12 & 0.15 & 1 & $3(3 \mathrm{~cm})$ & 3 & & LC-MS/MS & $\begin{array}{l}\text { Pichini } \\
\text { (2016) [33] }\end{array}$ \\
\hline & $0.83-1.8$ & & 1.4 & 1.6 & 1 & $3(1.5-2 \mathrm{~cm})$ & 3 & & GC-MS & Wille (2009) [25] \\
\hline & $0.034-2.0$ & & & $0.15-1.6$ & 8 & & 17 & & & Combined \\
\hline $\begin{array}{c}\text { Trazodone } \\
\text { (mCPP (M-chloro- } \\
\text { phenylpiperazine)) }\end{array}$ & $0.059-1.0$ & $0.088-0.50$ & 0.37 & 0.50 & 3 & $1-10(1 \mathrm{~cm})$ & 13 & $\begin{array}{c}\text { AD users, } \\
\text { presumed intake }\end{array}$ & LC-MS/MS & $\begin{array}{l}\text { Fernández } \\
\text { (2016) [19] }\end{array}$ \\
\hline \multirow{3}{*}{$\begin{array}{l}\text { Venlafaxine } \\
\text { (O-desmethyl- } \\
\text { venlafaxine) }\end{array}$} & - & $0.13-3.5$ & 1.5 & 0.92 & 45 & $2(2 \mathrm{~cm})$ or full length & $50(20)$ & \multirow{3}{*}{$\begin{array}{c}\text { PM case, } \\
\text { presumed intake } \\
\text { Headache } \\
\text { patients }\end{array}$} & LC-MS/MS & $\begin{array}{l}\text { Methling } \\
\text { (2020) [18] }\end{array}$ \\
\hline & $1.2-4.8$ & - & - & - & 6 & $1(3 \mathrm{~cm})$ & 6 & & LC-MS/MS & $\begin{array}{c}\text { Licata } \\
\text { (2016) [20] }\end{array}$ \\
\hline & $0.13-4.8$ & & & 0.92 & 51 & & $56(20)$ & & & Combined \\
\hline \multicolumn{11}{|l|}{ ANTIPSYCHOTICS } \\
\hline \multirow{3}{*}{$\begin{array}{c}\text { Aripiprazole } \\
\text { (Dehydroaripiprazole) }\end{array}$} & $0.21-1.5$ & $0.3-1.3$ & 0.76 & 0.72 & 16 & $1-6(1 \mathrm{~cm})$ & 71 & \multirow{3}{*}{$\begin{array}{l}\text { PM cases of } \\
\text { psychiatric } \\
\text { patients with } \\
\text { presumed intake } \\
\text { Schizophrenic } \\
\text { patients under } \\
\text { drug treatment, } \\
\text { in compliance }\end{array}$} & LC-MS/MS & $\begin{array}{l}\text { Rygaard } \\
(2020)[34]\end{array}$ \\
\hline & $0.030-0.093$ & $0.034-0.083$ & - & 0.050 & 9 & $1-2(2 \mathrm{~cm})$ & 11 & & LC-MS/MS & Wang (2019) [31] \\
\hline & $0.030-1.5$ & & & $0.050-0.72$ & 25 & & 82 & & & Combined \\
\hline
\end{tabular}


Table 1. Cont.

\begin{tabular}{|c|c|c|c|c|c|c|c|c|c|c|}
\hline $\begin{array}{c}\text { Pharmaceutical } \\
\text { (Metabolite) }\end{array}$ & Range & $\begin{array}{l}\text { MD Ratio } \\
\text { 10-90 } \text { PCTL }^{(\mathrm{A})} \\
\end{array}$ & Mean & Median & No. of Subjects & $\begin{array}{l}\text { No. of Seg. Per Subject } \\
\text { (Seg. Length) }\end{array}$ & $\begin{array}{l}\text { Total No. of Seg. } \\
\text { (Whole Strand) }\end{array}$ & $\begin{array}{c}\text { Case } \\
\text { Description }\end{array}$ & $\begin{array}{l}\text { Analytical } \\
\text { Method }\end{array}$ & Reference \\
\hline \multirow{3}{*}{$\begin{array}{l}\text { Chlorprothixene } \\
\text { (N-desmethyl- } \\
\text { chlorprothixene) }\end{array}$} & - & $0.41-1.5$ & - & 0.93 & 20 & $3-6(1 \mathrm{~cm})$ & $\geq 60$ & $\begin{array}{c}\text { PM cases of } \\
\text { psychiatric } \\
\text { patients with } \\
\text { presumed intake }\end{array}$ & LC-MS/MS & $\begin{array}{l}\text { Günther } \\
\text { (2018) [35] }\end{array}$ \\
\hline & 1.5 & - & 1.5 & - & 1 & $1(2 \mathrm{~cm})$ & 1 & $\begin{array}{l}\text { Suspected DFC } \\
\text { case }\end{array}$ & LC-MS/MS & Wang (2018) [28] \\
\hline & $0.41-1.5$ & & - & 0.93 & 21 & & $\geq 61$ & & & Combined \\
\hline \multirow[b]{3}{*}{$\begin{array}{c}\text { Clozapine } \\
\text { (N-desmethylclozapine) }\end{array}$} & - & $0.075-1.3^{(\mathrm{B})}$ & 0.57 & 0.52 & 25 & $2(2 \mathrm{~cm})$ or full length & $28(11)$ & \multirow{3}{*}{$\begin{array}{c}\text { PM cases } \\
\text { presumed intake } \\
\text { Schizophrenic } \\
\text { patients under } \\
\text { drug treatment, } \\
\text { in compliance } \\
\text { Patients in } \\
\text { low-dose } \\
\text { treatment, } \\
\text { pigmented hair } \\
\text { and } \\
\text { non-pigmented } \\
\text { hair }\end{array}$} & LC-MS/MS & $\begin{array}{l}\text { Methling } \\
\text { (2020) [18] }\end{array}$ \\
\hline & $0.52-1.5$ & $0.77-1.4$ & - & 1.1 & 27 & $1-3(2 \mathrm{~cm})$ & 54 & & LC-MS/MS & Wang (2019) [31] \\
\hline & $\begin{array}{l}0.97-1.6 \\
0.70-1.3\end{array}$ & $\begin{array}{l}1.0-1.6 \\
0.75-1.1\end{array}$ & $\begin{array}{c}1.2 \\
0.96\end{array}$ & $\begin{array}{c}1.2 \\
0.97\end{array}$ & $\begin{array}{l}12 \\
11\end{array}$ & $\begin{array}{l}1(2-7 \mathrm{~cm}) \\
1(2-7 \mathrm{~cm})\end{array}$ & $\begin{array}{l}12 \\
11\end{array}$ & & LC-MS/MS & $\begin{array}{l}\text { Kronstrand } \\
\text { (2007) [36] }\end{array}$ \\
\hline \multirow{3}{*}{$\begin{array}{c}\text { Olanzapine } \\
(N- \\
\text { desmethylolanzapine })\end{array}$} & $0.010-3.4$ & - & - & 0.59 & 34 & $\begin{array}{l}1-6(1 \mathrm{~cm}) \text { or full length } \\
\text { (if }<2 \mathrm{~cm})\end{array}$ & 105 & $\begin{array}{c}\text { PM cases of } \\
\text { psychiatric } \\
\text { patients, } \\
\text { presumed intake }\end{array}$ & LC-MS/MS & $\begin{array}{l}\text { Günter } \\
\text { (2020) [37] }\end{array}$ \\
\hline & $0.063-0.23$ & $0.063-0.23$ & - & 0.093 & 5 & $1-2(2 \mathrm{~cm})$ & 7 & $\begin{array}{l}\text { patients under } \\
\text { drug treatment, } \\
\text { in compliance }\end{array}$ & LC-MS/MS & Wang (2019) [31] \\
\hline & $0.010-3.4$ & & & $0.093-0.59$ & 39 & & 112 & & & Combined \\
\hline \multirow{4}{*}{$\begin{array}{c}\text { Risperidone } \\
\text { (9-OH-risperidone) }\end{array}$} & $0.0045-0.093$ & $0.0055-0.031$ & - & 0.014 & 12 & $1-3(2 \mathrm{~cm})$ & 27 & $\begin{array}{l}\text { Schizophrenic } \\
\text { patients under } \\
\text { drug treatment, } \\
\text { in compliance }\end{array}$ & LC-MS/MS & Wang (2019) [31] \\
\hline & - & $0.0047-1^{(\mathrm{B})}$ & 0.17 & 0.058 & 35 & $2(2 \mathrm{~cm})$ or full length & $30(20)$ & $\begin{array}{l}\text { PM cases, } \\
\text { presumed intake }\end{array}$ & LC-MS/MS & $\begin{array}{l}\text { Methling } \\
\text { (2020) [18] }\end{array}$ \\
\hline & $0.012-1.0$ & - & 0.31 & 0.023 & 3 & $1-4(2-10 \mathrm{~cm})$ & 8 & $\begin{array}{l}\text { Psychiatric } \\
\text { patients }\end{array}$ & LC-MS/MS & $\begin{array}{l}\text { Schneider } \\
\text { (2009) [38] }\end{array}$ \\
\hline & $0.0045-1.0$ & & & $0.014-0.058$ & 50 & & $65(20)$ & & & Combined \\
\hline
\end{tabular}


Table 1. Cont.

\begin{tabular}{|c|c|c|c|c|c|c|c|c|c|c|}
\hline $\begin{array}{c}\text { Pharmaceutical } \\
\text { (Metabolite) }\end{array}$ & Range & $\begin{array}{l}\text { MD Ratio } \\
\text { 10-90 } \text { PCTL }^{\text {(A) }}\end{array}$ & Mean & Median & No. of Subjects & $\begin{array}{l}\text { No. of Seg. Per Subject } \\
\text { (Seg. Length) }\end{array}$ & $\begin{array}{l}\text { Total No. of Seg. } \\
\text { (Whole Strand) }\end{array}$ & $\begin{array}{c}\text { Case } \\
\text { Description }\end{array}$ & $\begin{array}{l}\text { Analytical } \\
\text { Method }\end{array}$ & Reference \\
\hline $\begin{array}{c}\text { Quetiapine } \\
\text { (7-OH-quetiapine) }\end{array}$ & $0.074-1$ & $0.12-0.91$ & 0.50 & 0.49 & 10 & $1-6(2 \mathrm{~cm})$ & 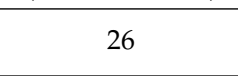 & $\begin{array}{l}\text { Patients under } \\
\text { drug treatment }\end{array}$ & LC-MS/MS & Binz (2014) [39] \\
\hline \multicolumn{11}{|l|}{ BENZODIAZEPINES } \\
\hline \multirow{6}{*}{$\begin{array}{c}\text { Clonazepam } \\
\text { (7-aminoclonazepam) }\end{array}$} & $0.16-17$ & - & - & 2.8 & 33 & $1(\leq 5 \mathrm{~cm})$ & 33 & $\begin{array}{c}\text { Individuals } \\
\text { undergoing } \\
\text { toxicological } \\
\text { investigation }\end{array}$ & LC-MS/MS & $\begin{array}{l}\text { Madry } \\
(2020)[41]\end{array}$ \\
\hline & 82 & - & 82 & - & 1 & Unspecified & $\geq 1$ & $\begin{array}{l}\text { Suspected drug } \\
\text { abuser }\end{array}$ & LC-MS/MS & Shin (2019) [42] \\
\hline & 1.6 & - & 1.6 & - & 1 & $1(2 \mathrm{~cm})$ & 1 & $\begin{array}{l}\text { Suspected DFC } \\
\text { case }\end{array}$ & LC-MS/MS & Wang (2018) [28] \\
\hline & 3.7 & - & 3.7 & - & 1 & $1(3 \mathrm{~cm})$ & 1 & $\begin{array}{c}\text { Headache } \\
\text { patient }\end{array}$ & LC-MS/MS & $\begin{array}{c}\text { Licata } \\
\text { (2016) [20] }\end{array}$ \\
\hline & 22 & - & 22 & - & 1 & Unspecified & $\geq 1$ & DFC case & LC-MS/MS & Chéze 2005 [43] \\
\hline & 7.0 & - & 7.0 & - & 1 & $1(2 \mathrm{~cm})$ & 1 & $\begin{array}{c}\text { Individuals } \\
\text { under treatment }\end{array}$ & GC-MS & $\begin{array}{c}\text { Negruz } \\
(2000)[44]\end{array}$ \\
\hline \multirow{6}{*}{$\begin{array}{c}\text { Diazepam } \\
\text { (Nordiazepam) }\end{array}$} & $0.0056-26$ & - & - & 1.6 & 293 & $1(\leq 5 \mathrm{~cm})$ & 293 & $\begin{array}{c}\text { Individuals } \\
\text { undergoing } \\
\text { toxicological } \\
\text { investigation }\end{array}$ & LC-MS/MS & $\begin{array}{l}\text { Madry } \\
(2020)[41]\end{array}$ \\
\hline & - & $0.26-2.9^{(\mathrm{B})}$ & 1.3 & 1.1 & 71 & $2(2 \mathrm{~cm})$ or full length & $70(36)$ & $\begin{array}{c}\text { PM cases, } \\
\text { presumed intake }\end{array}$ & LC-MS/MS & $\begin{array}{l}\text { Methling } \\
\text { (2020) [18] }\end{array}$ \\
\hline & 0.40 & - & 0.40 & - & 1 & Unspecified & $\geq 1$ & $\begin{array}{l}\text { Individuals with } \\
\text { suspected drug } \\
\text { abuse }\end{array}$ & LC-MS/MS & Shin (2019) [42] \\
\hline & 0.96 & - & 0.96 & - & 1 & $1(3-6 \mathrm{~cm})$ & 1 & $\begin{array}{l}\text { Driving licence } \\
\text { regranting }\end{array}$ & LC-MS/MS & $\begin{array}{l}\text { Lendoiro } \\
\text { (2018) [45] }\end{array}$ \\
\hline & $0.58-1.6$ & - & 1.0 & 0.98 & 2 & $1-3(0.8-1.5 \mathrm{~cm})$ & 4 & $\begin{array}{l}\text { Suspected DFC } \\
\text { case }\end{array}$ & LC-MS/MS & Wang (2018) [28] \\
\hline & 0.98 & - & 0.98 & - & 1 & $1(3-6 \mathrm{~cm})$ & 1 & PM case & LC-MS/MS & $\begin{array}{c}\text { Morini } \\
\text { (2017) [46] }\end{array}$ \\
\hline
\end{tabular}


Table 1. Cont.

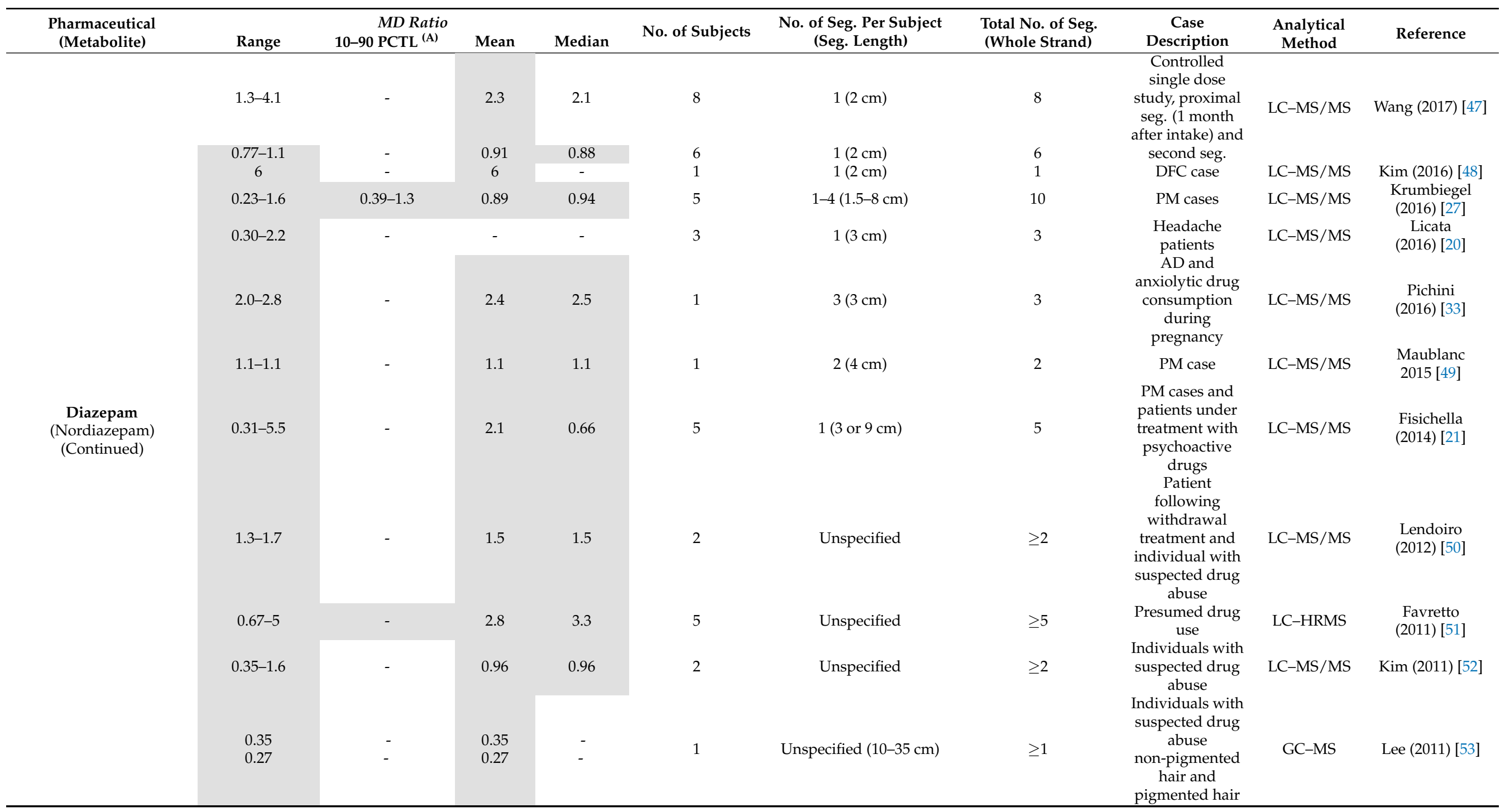


Table 1. Cont.

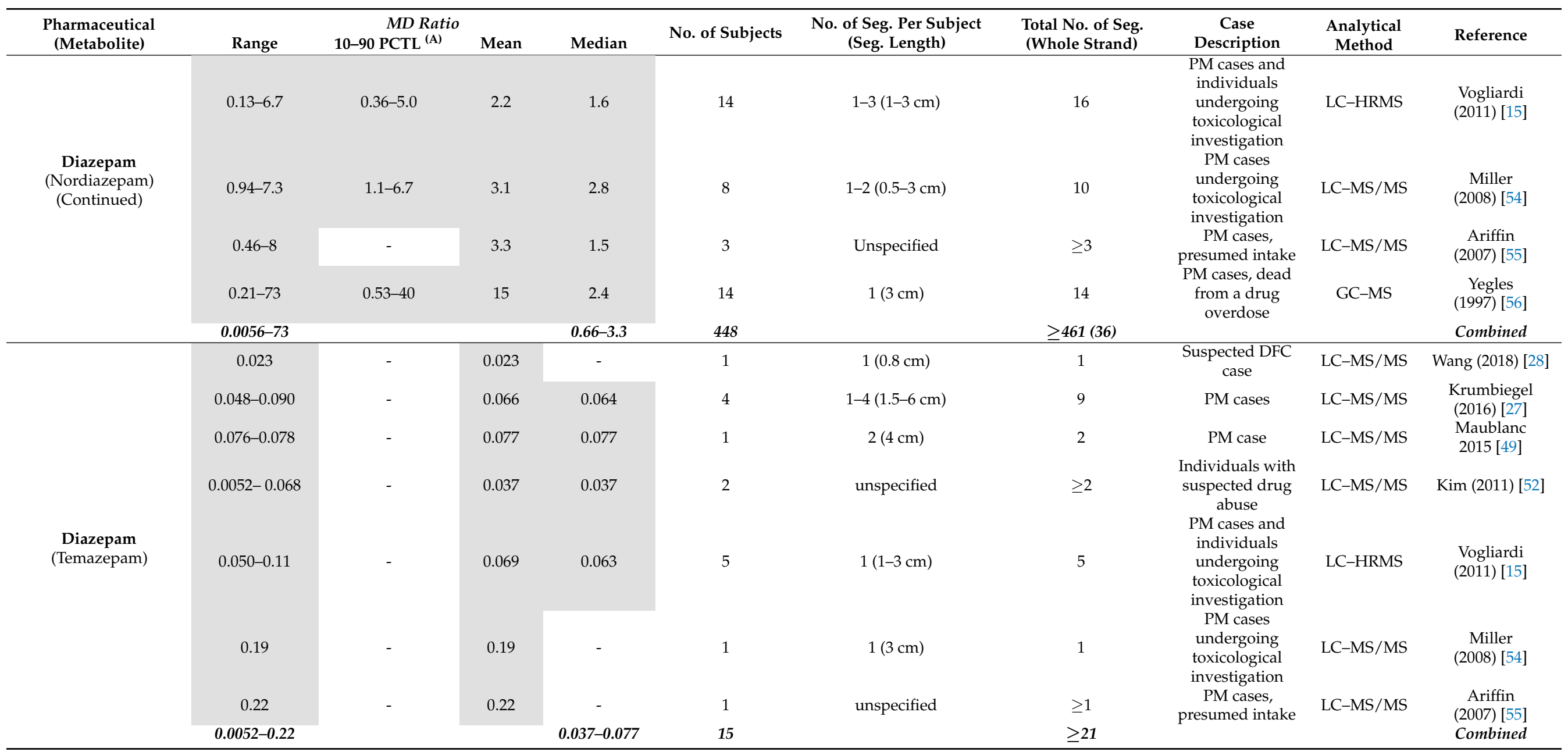


Table 1. Cont

\begin{tabular}{|c|c|c|c|c|c|c|c|c|c|c|}
\hline $\begin{array}{l}\text { Pharmaceutical } \\
\text { (Metabolite) }\end{array}$ & Range & $\begin{array}{l}\text { MD Ratio } \\
\text { 10-90 } \text { PCTL }^{\text {(A) }}\end{array}$ & Mean & Median & No. of Subjects & $\begin{array}{l}\text { No. of Seg. Per Subject } \\
\text { (Seg. Length) }\end{array}$ & $\begin{array}{l}\text { Total No. of Seg. } \\
\text { (Whole Strand) }\end{array}$ & $\begin{array}{c}\text { Case } \\
\text { Description }\end{array}$ & $\begin{array}{l}\text { Analytical } \\
\text { Method }\end{array}$ & Reference \\
\hline \multirow{7}{*}{$\begin{array}{c}\text { Flunitrazepam } \\
\text { (7-amino-flunitrazepam) }\end{array}$} & $2.1-20$ & - & - & 6.5 & 8 & $1(\leq 5 \mathrm{~cm})$ & 8 & $\begin{array}{l}\text { Individuals } \\
\text { undergoing } \\
\text { toxicological } \\
\text { investigation }\end{array}$ & LC-MS/MS & $\begin{array}{l}\text { Madry } \\
(2020)[41]\end{array}$ \\
\hline & - & $1.3-5.0^{(\mathrm{B})}$ & 3.2 & 3.1 & 3 & $2(2 \mathrm{~cm})$ or full length & $4(1)$ & $\begin{array}{l}\text { PM cases, } \\
\text { presumed intake }\end{array}$ & LC-MS/MS & $\begin{array}{l}\text { Methling } \\
(2020)[18]\end{array}$ \\
\hline & - & $0.51-8.3$ & - & 3.07 & 22 & $3-6(1 \mathrm{~cm})$ & 76 & $\begin{array}{l}\text { Individuals } \\
\text { undergoing } \\
\text { toxicological } \\
\text { investigation, } \\
\text { repeated use }\end{array}$ & LC-MS/MS & Zhuo (2020) [9] \\
\hline & 1.1 & - & 1.1 & - & 1 & $1(3 \mathrm{~cm})$ & 1 & DFC case & LC-MS/MS & $\operatorname{Kim}(2016)$ [48] \\
\hline & 1.1 & - & 1.1 & - & 1 & unspecified & $\geq 1$ & PM case & GC-MS & $\begin{array}{c}\text { Negrusz } \\
\text { (1999) [57] }\end{array}$ \\
\hline & $0.069-3.5$ & $0.27-2.5$ & 1.2 & 0.90 & 14 & Whole strand & (14) & $\begin{array}{c}\text { PM cases, } \\
\text { polydrug } \\
\text { abusers }\end{array}$ & GC-MS & $\begin{array}{l}\text { Cirimele } \\
\text { (1997) [58] }\end{array}$ \\
\hline & $0.11-0.27$ & - & 0.18 & 0.17 & 1 & $3(3-4 \mathrm{~cm})$ & 3 & $\begin{array}{l}\text { Individual with } \\
\text { a suspected } \\
\text { drug abuse }\end{array}$ & GC-MS & $\begin{array}{l}\text { Cirimele } \\
\text { (1996) [59] }\end{array}$ \\
\hline $\begin{array}{c}\text { Flurazepam } \\
\text { (2-hydroxyethyl- } \\
\text { flurazepam) }\end{array}$ & 0.033 & - & 0.033 & - & 1 & $1(3 \mathrm{~cm})$ & 1 & $\begin{array}{l}\text { Headache } \\
\text { patients }\end{array}$ & LC-MS/MS & $\begin{array}{c}\text { Licata } \\
(2016)[20]\end{array}$ \\
\hline \multirow{3}{*}{$\begin{array}{c}\text { Flurazepam } \\
\text { (N-desalkylflurazepam) }\end{array}$} & $0.60-110$ & - & - & 10 & 52 & $1(\leq 5 \mathrm{~cm})$ & 52 & $\begin{array}{c}\text { Individuals } \\
\text { undergoing } \\
\text { toxicological } \\
\text { investigation }\end{array}$ & LC-MS/MS & $\begin{array}{l}\text { Madry } \\
(2020)[41]\end{array}$ \\
\hline & 2.4 & - & 2.4 & - & 1 & $1(3 \mathrm{~cm})$ & 1 & $\begin{array}{l}\text { Headache } \\
\text { patients }\end{array}$ & LC-MS/MS & $\begin{array}{c}\text { Licata } \\
\text { (2016) [20] }\end{array}$ \\
\hline & $0.60-110$ & & & 10 & 53 & & 53 & & & Combined \\
\hline \multirow[t]{3}{*}{$\begin{array}{l}\text { Lormetazepam } \\
\text { (Lorazepam) }\end{array}$} & $0.18-8$ & - & - & - & 8 & $1(\leq 5 \mathrm{~cm})$ & 8 & $\begin{array}{c}\text { Individuals } \\
\text { undergoing } \\
\text { toxicological } \\
\text { investigation }\end{array}$ & LC-MS/MS & $\begin{array}{l}\text { Madry } \\
(2020)[41]\end{array}$ \\
\hline & 0.42 & - & 0.42 & - & 1 & $1(3 \mathrm{~cm})$ & 1 & $\begin{array}{l}\text { Headache } \\
\text { patients }\end{array}$ & LC-MS/MS & $\begin{array}{c}\text { Licata } \\
\text { (2016) [20] }\end{array}$ \\
\hline & $0.18-8$ & & & - & 9 & & 9 & & & Combined \\
\hline
\end{tabular}


Table 1. Cont.

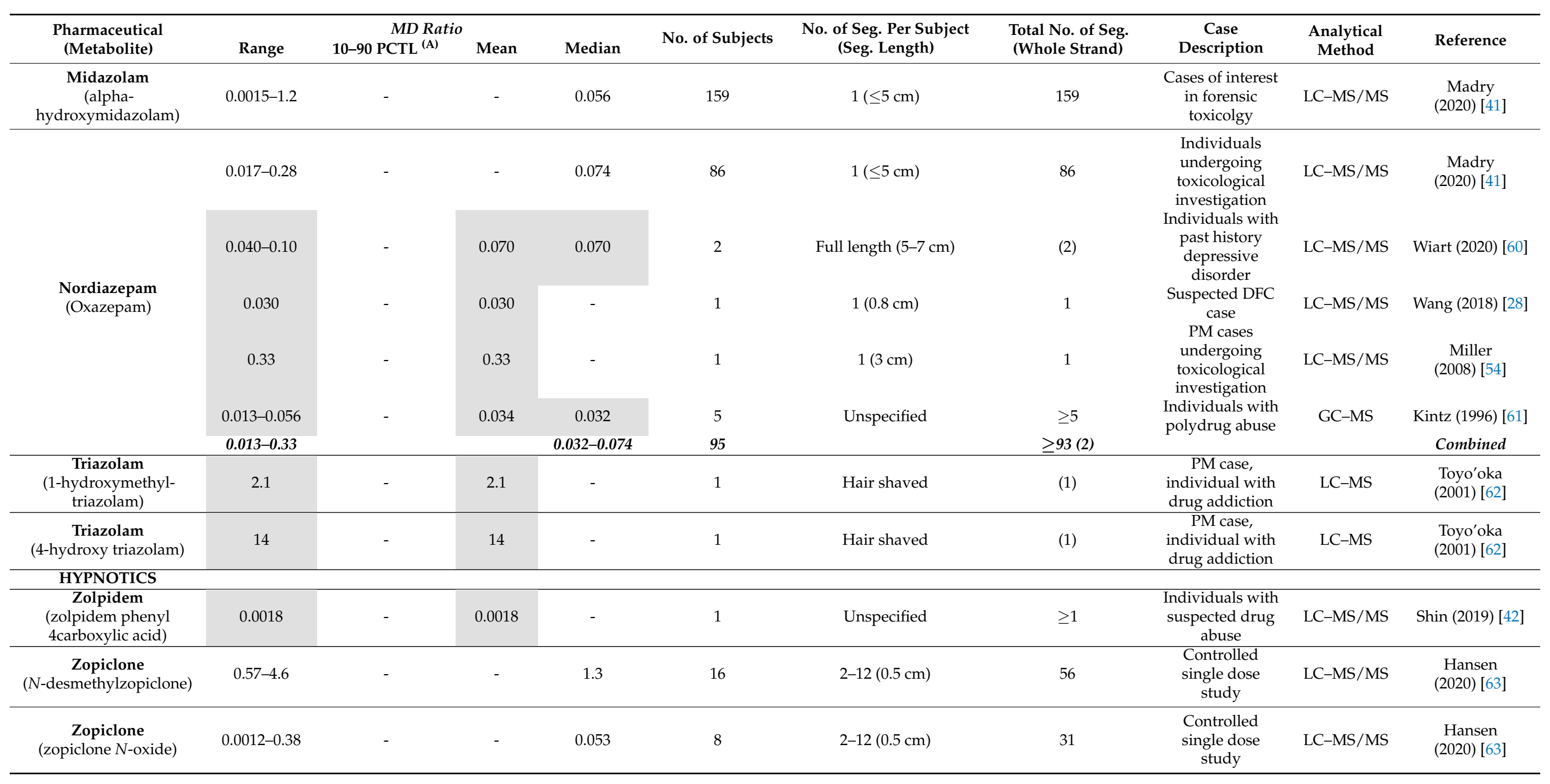


Table 1. Cont

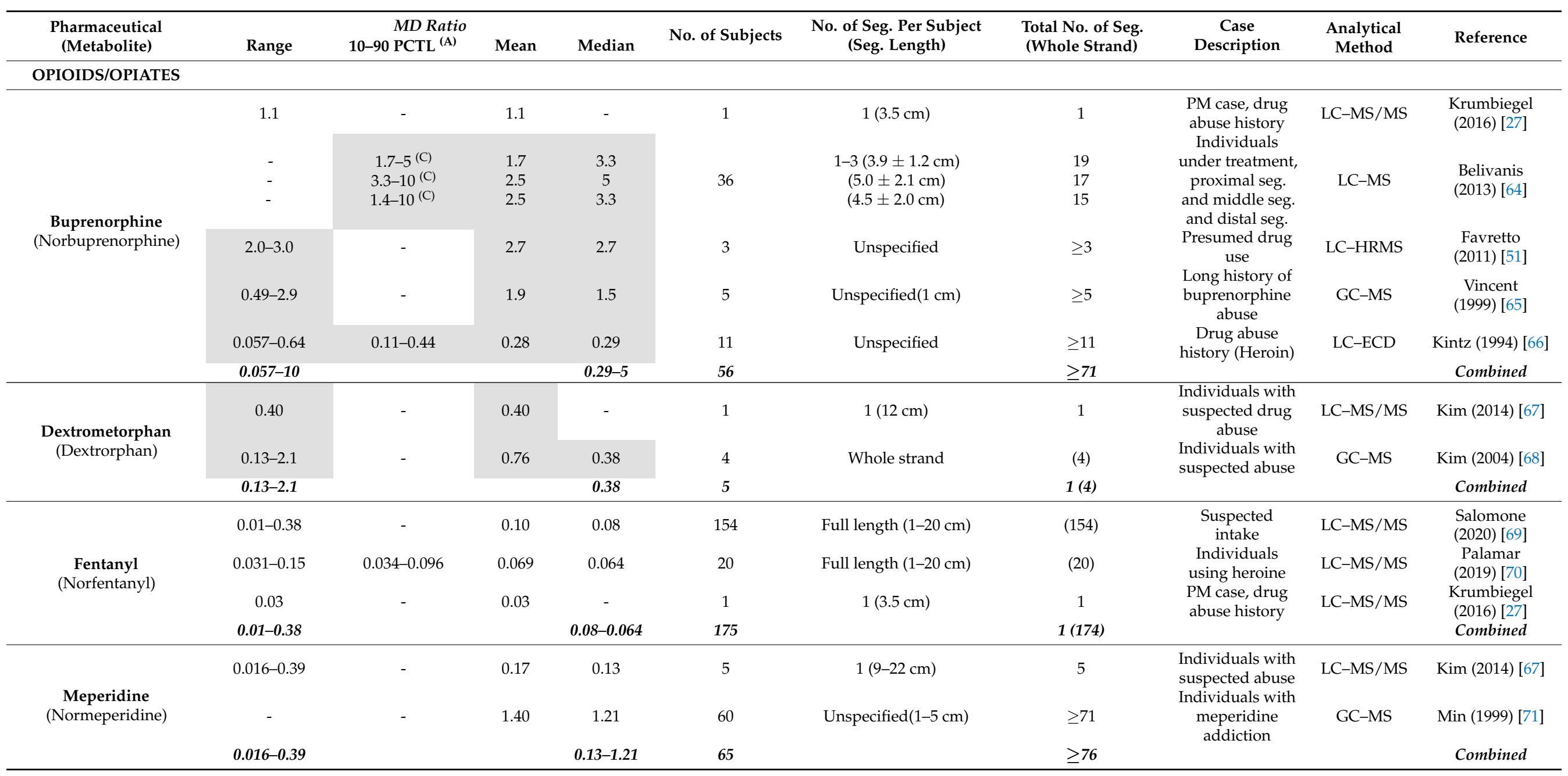


Table 1. Cont

\begin{tabular}{|c|c|c|c|c|c|c|c|c|c|c|}
\hline $\begin{array}{l}\text { Pharmaceutical } \\
\text { (Metabolite) }\end{array}$ & Range & $\begin{array}{l}\text { MD Ratio } \\
\text { 10-90 } \text { PCTL }^{\text {(A) }}\end{array}$ & Mean & Median & No. of Subjects & $\begin{array}{l}\text { No. of Seg. Per Subject } \\
\text { (Seg. Length) }\end{array}$ & $\begin{array}{l}\text { Total No. of Seg. } \\
\text { (Whole Strand) }\end{array}$ & $\begin{array}{c}\text { Case } \\
\text { Description }\end{array}$ & $\begin{array}{l}\text { Analytical } \\
\text { Method }\end{array}$ & Reference \\
\hline \multirow{15}{*}{$\begin{array}{c}\text { Methadone } \\
\text { (EDDP } \\
\text { (2-ethyl-1,5-dimethyl- } \\
\text { 3,3- } \\
\text { diphenylpyrrolidine)) }\end{array}$} & 0.30 & - & 0.30 & - & 1 & Full length & (1) & $\begin{array}{l}\text { Individual } \\
\text { undergoing } \\
\text { methadone } \\
\text { treatment } \\
\text { program }\end{array}$ & $\begin{array}{l}\text { GC- } \\
\text { MS/MS }\end{array}$ & $\begin{array}{l}\text { Rosado } \\
(2020)[72]\end{array}$ \\
\hline & 0.063 & - & 0.063 & - & 5 & $1(3 \mathrm{~cm})$ & 5 & $\begin{array}{l}\text { Patients in } \\
\text { treatment for } \\
\text { substance-use } \\
\text { disorders }\end{array}$ & LC-MS/MS & $\begin{array}{l}\text { Cappelle } \\
(2018)[73]\end{array}$ \\
\hline & 0.063 & - & 0.063 & - & 1 & Unspecified & $\geq 1$ & $\begin{array}{l}\text { Individuals with } \\
\text { self-reported } \\
\text { drug use }\end{array}$ & LC-MS/MS & $\begin{array}{l}\text { Cappelle } \\
\text { (2017) [74] }\end{array}$ \\
\hline & $0.11-0.15$ & - & 0.13 & 0.12 & 1 & $4(1 \mathrm{~cm})$ & 4 & Child sedated & LC-MS/MS & Kintz (2017) [75] \\
\hline & $0.060-0.099$ & - & 0.075 & 0.074 & 3 & $1-4(2-6 \mathrm{~cm})$ & 6 & $\begin{array}{c}\text { PM cases, } \\
\text { known intake }\end{array}$ & LC-MS/MS & $\begin{array}{c}\text { Krumbiegel } \\
\text { (2016) [27] }\end{array}$ \\
\hline & 1 & - & 1 & - & 1 & $1(3 \mathrm{~cm})$ & 1 & $\begin{array}{l}\text { Maternal hair } \\
\text { sample }\end{array}$ & LC-MS/MS & Joya (2015) [76] \\
\hline & 0.035 & - & 0.035 & - & 1 & $1(10 \mathrm{~cm})$ & 1 & $\begin{array}{l}\text { Psychiatric } \\
\text { patient }\end{array}$ & LC-MS/MS & $\begin{array}{l}\text { Koster } \\
\text { (2014) [77] }\end{array}$ \\
\hline & $0.013-0.39$ & - & 0.15 & 0.043 & 1 & $3(1.5 \mathrm{~cm})$ & 3 & $\begin{array}{c}\text { Maternal hair } \\
\text { samples, seg. } \\
2-4\end{array}$ & LC-MS/MS & $\begin{array}{l}\text { Tournel } \\
\text { (2014) [78] }\end{array}$ \\
\hline & $0.037-0.48$ & - & 0.23 & 0.19 & 7 & $1(5-7 \mathrm{~cm})$ & 7 & $\begin{array}{c}\text { Cases } \\
\text { undergoing } \\
\text { toxicological } \\
\text { investigation, } \\
\text { presumed drug } \\
\text { use }\end{array}$ & LC-HRMS & $\begin{array}{l}\text { Favretto } \\
\text { (2014) [79] }\end{array}$ \\
\hline & $0.26-0.53$ & - & 0.40 & 0.40 & 2 & unspecified & $\geq 2$ & $\begin{array}{c}\text { Presumed drug } \\
\text { use }\end{array}$ & LC-HRMS & $\begin{array}{l}\text { Favretto } \\
(2011)[51]\end{array}$ \\
\hline & $0.1-0.3$ & - & 0.2 & 0.2 & 9 & $1(3 \mathrm{~cm})$ & 9 & $\begin{array}{c}\text { Methadone } \\
\text { maintenance } \\
\text { therapy }\end{array}$ & GC-MS & Fucci (2007) [80] \\
\hline & $0.099-0.23$ & - & 0.16 & 0.16 & 7 & Whole strand & (7) & $\begin{array}{l}\text { Methadone } \\
\text { maintenance } \\
\text { therapy }\end{array}$ & GC-MS & Lucas (2000) [81] \\
\hline & $0.19-0.67$ & - & 0.39 & - & 17 & $1(3 \mathrm{~cm})$ & 17 & $\begin{array}{l}\text { PM cases, died } \\
\text { from overdose }\end{array}$ & GC-MS & $\begin{array}{l}\text { Sporkert } \\
(2000)[82]\end{array}$ \\
\hline & $\begin{array}{c}0.14-0.67 \\
0.14-0.19 \\
0.031-0.71\end{array}$ & $\begin{array}{l}- \\
- \\
-\end{array}$ & $\begin{array}{c}0.18 \\
0.14 \\
0.062\end{array}$ & $\begin{array}{l}- \\
- \\
-\end{array}$ & 3 & $10-29(2 \mathrm{~cm})$ & $\begin{array}{l}29 \\
10 \\
23\end{array}$ & $\begin{array}{l}\text { Methadone } \\
\text { maintenance } \\
\text { therapy }\end{array}$ & GC-MS & $\begin{array}{l}\text { Moeller } \\
\text { (1993) [83] }\end{array}$ \\
\hline & $0.013-0.71$ & & & $0.043-0.40$ & 59 & & $118(8)$ & & & Combined \\
\hline
\end{tabular}


Table 1. Cont.

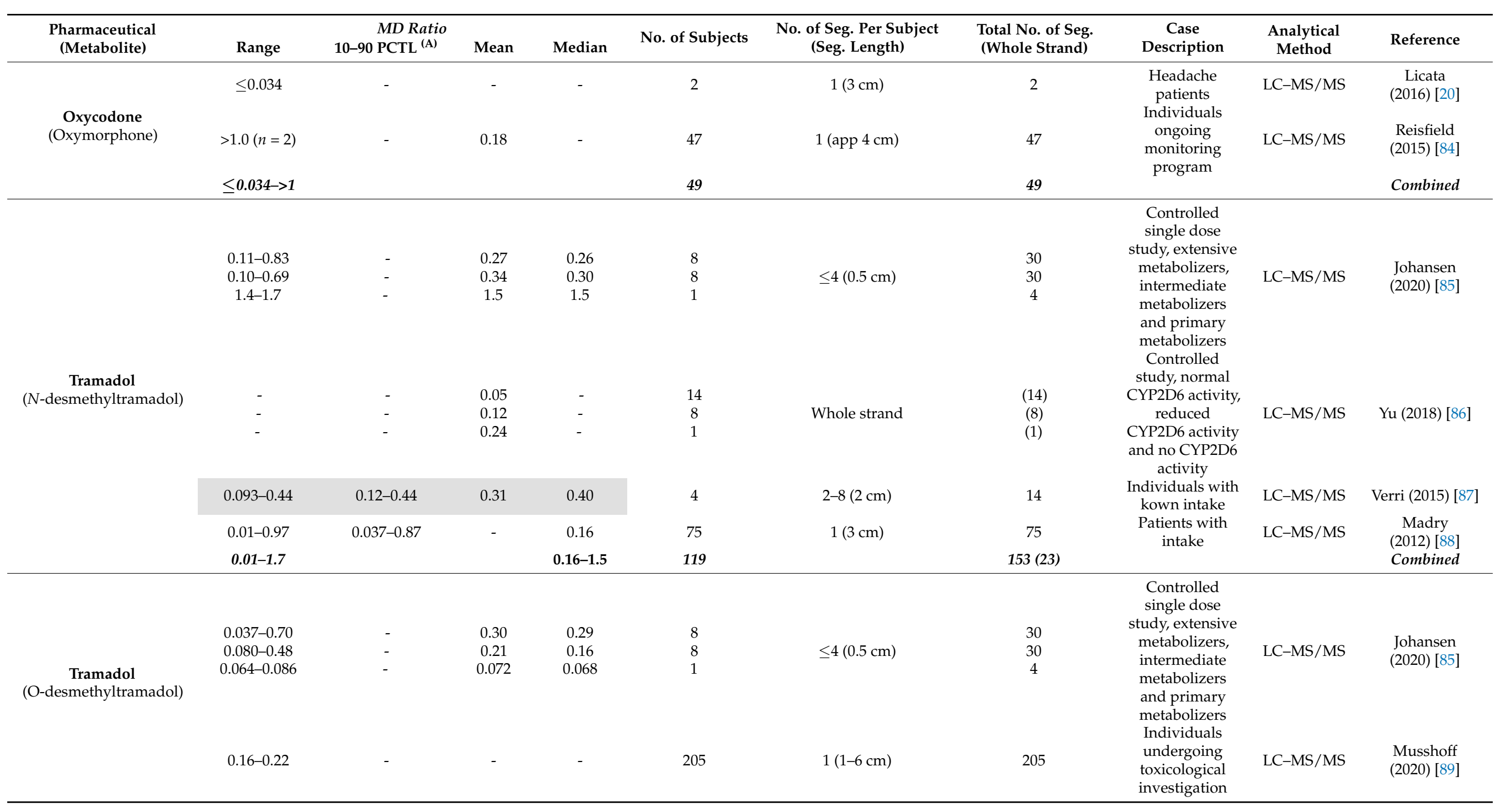


Table 1. Cont.

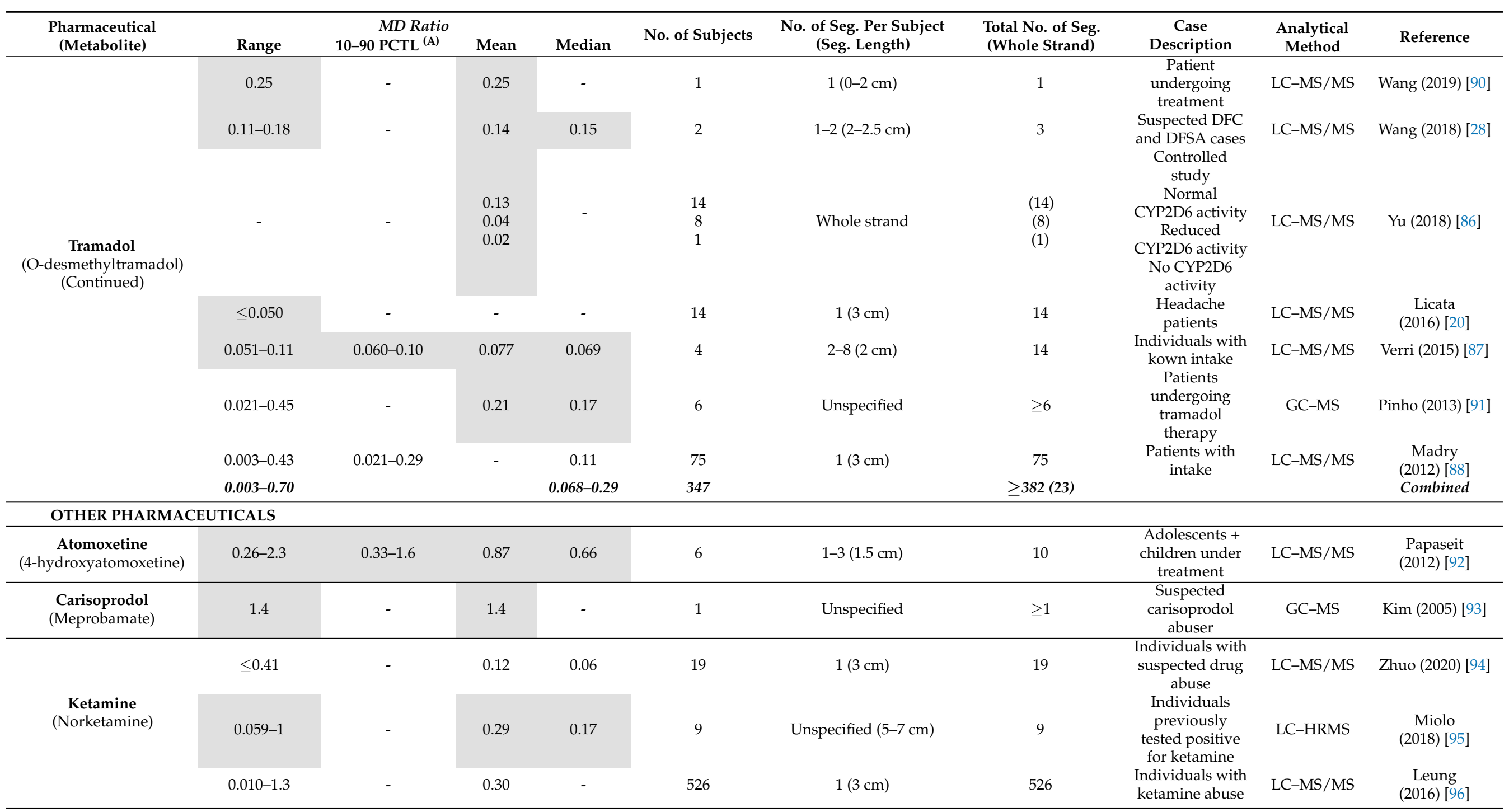


Table 1. Cont.

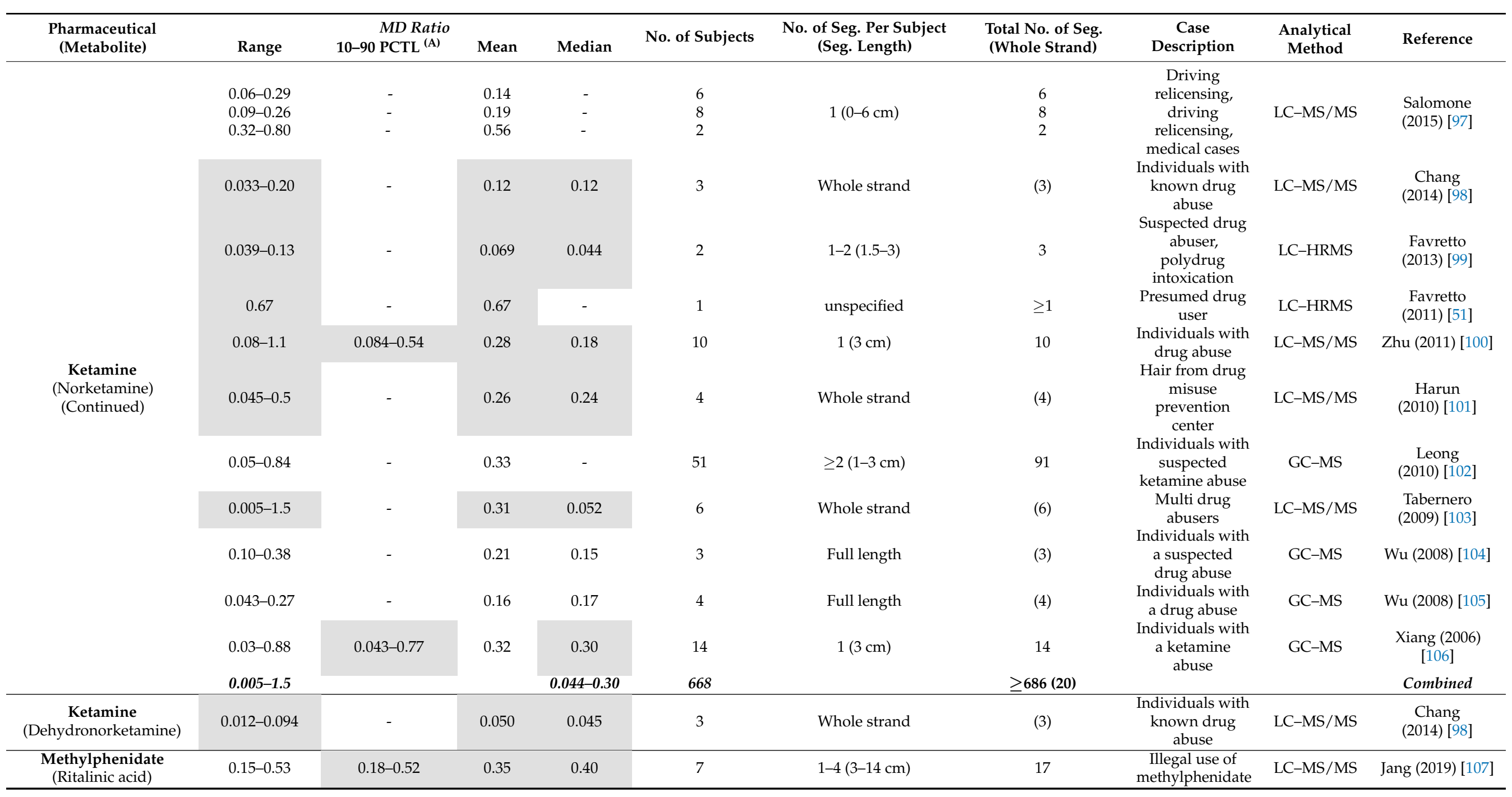


Table 1. Cont.

\begin{tabular}{|c|c|c|c|c|c|c|c|c|c|c|}
\hline $\begin{array}{l}\text { Pharmaceutical } \\
\text { (Metabolite) }\end{array}$ & Range & $\begin{array}{r}\text { MD Ratio } \\
\text { 10-90 } \text { PCTL }^{(\mathrm{A})}\end{array}$ & Mean & Median & No. of Subjects & $\begin{array}{l}\text { No. of Seg. Per Subject } \\
\text { (Seg. Length) }\end{array}$ & $\begin{array}{l}\text { Total No. of Seg. } \\
\text { (Whole Strand) }\end{array}$ & $\begin{array}{c}\text { Case } \\
\text { Description }\end{array}$ & $\begin{array}{l}\text { Analytical } \\
\text { Method }\end{array}$ & Reference \\
\hline \multirow{3}{*}{$\begin{array}{c}\text { Oxcarbazepine } \\
(10- \\
\text { hydroxycarbamazepine) }\end{array}$} & $0.39-0.64$ & 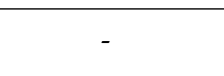 & 0.51 & 0.50 & 1 & $3(2 \mathrm{~cm})$ & ( & $\begin{array}{l}\text { PM case, drug } \\
\text { addiction }\end{array}$ & LC-MS/MS & Wang 2017 [40] \\
\hline & $4.7-8.1$ & - & 6.0 & 5.2 & 1 & $3(2 \mathrm{~cm})$ & 3 & $\begin{array}{c}\text { PM case, } \\
\text { psychiatric } \\
\text { patient in } \\
\text { the past }\end{array}$ & LC-MS & Klys (2005) [108 \\
\hline & $0.39-8.1$ & & & $0.50-5.2$ & 2 & & 6 & & & Combined \\
\hline $\begin{array}{c}\text { Oxcarbazepine } \\
\text { (Trans-diol-carbazepine) }\end{array}$ & $0.12-0.23$ & - & 0.16 & 0.13 & 1 & $3(2 \mathrm{~cm})$ & 3 & $\begin{array}{c}\text { PM case, } \\
\text { psychiatric } \\
\text { patient in } \\
\text { the past }\end{array}$ & LC-MS & Klys (2005) [108 \\
\hline $\begin{array}{c}\text { Thiopental } \\
\text { (Pentobarbital) }\end{array}$ & $1.0-1.3$ & - & 1.2 & 1.3 & 1 & $3(1.5)$ & 3 & $\begin{array}{l}\text { DFSA, proximal } \\
\text { segments } \\
\text { analyzed }\end{array}$ & $\begin{array}{c}\text { GC- } \\
\mathrm{MS} / \mathrm{MS}\end{array}$ & $\begin{array}{c}\text { Frison } \\
\text { (2003) [109] }\end{array}$ \\
\hline
\end{tabular}

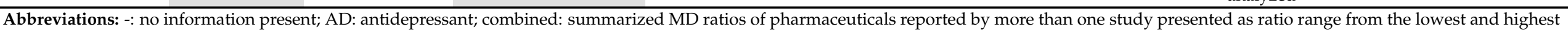

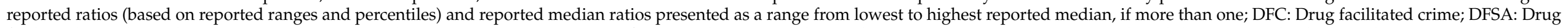

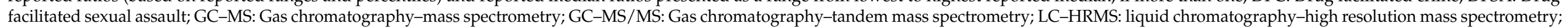

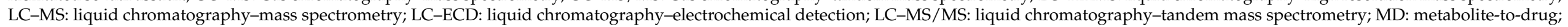
no.: number; PCTL: percentile; PM: postmortem; seg.: segments. (A) If only percentiles were reported in a study, this was used as range (B) 5-95 percentile, (C) 25-75 percentile. 
Figure 2 illustrates the wide-ranging MD ratios of commonly reported pharmaceuticals based on the lowest and the highest reported values in the literature. In addition, it shows that median MD ratios below 1 were frequently reported, indicating that the parent drug concentration in hair exceeds that of its metabolites. There may be several explanations behind MD ratios below 1 being frequently found, including the differences in the incorporation into hair or the metabolism of the parent pharmaceutical relative to its metabolite. Various mechanisms for drugs entering the hair have been proposed and the properties of the incorporated drugs and metabolites, as well as the physical and physiological characteristics of the individual, may strongly influence which mechanism will dominate and thus the amount incorporated, the incorporation site and consequently the MD ratio [3]. The properties of the drugs and metabolites affecting the incorporation into hair include lipophilicity $(\log \mathrm{P})$, the basicity $(\mathrm{pKa})$, melanin affinity, the half-life of elimination, and blood concentration. In general, lipophilic and basic compounds are better accumulated in pigmented hair than acidic compounds, which have been found in lower concentrations in hair [3]. This can explain the MD ratios less than $0.53(n=10$, see Table 1$)$ reported for methylphenidate and its more acidic metabolite ritalinic acid. MD ratios below 1 were primarily reported for several pharmaceuticals, including fentanyl, ketamine, methadone, and tramadol. This is in accordance with the higher parent drug concentrations reported for these pharmaceuticals by Musshoff et al. [89]. Similar physical and chemical properties were observed for many pharmaceuticals and their metabolites, as being the case for amitriptyline with pKa 9.4 and $\log \mathrm{P} 4.9$ and its metabolite nortriptyline with pKa 9.7 and $\log P 3.9$ [110], and thus, the MD ratios were not found to be strongly affected by the small differences in lipophilicity or pKa values. Yet, the formation of metabolites by demethylation leading to decreased lipophilicity of the metabolite compared with the parent pharmaceutical can point to less effective incorporation and thus MD ratios below 1 . This applies to most of the antidepressant pharmaceuticals, especially citalopram, doxepine, imipramine, and sertraline and for the antipsychotic pharmaceuticals, especially clozapine and olanzapine. In contrast, in plasma, the steady state concentrations of these metabolites have been found to be rather equal or higher than of the parent pharmaceuticals, giving MD ratios equal to or above 1 in plasma [23]. However, MD ratios in plasma and other fluid matrices are dependent on sampling and dosing time and represent a snapshot of the drug metabolism, which should always be considered. This contrasts MD ratios in hair representing the cumulative amount and the entire time course of drug metabolism [111].

MD ratios in hair above 1 were primarily reported for amitriptyline, buprenorphine, clomipramine, clonazepam, diazepam (with metabolite nordiazepam), flunitrazepam, flurazepam, oxcarbazepine (with metabolite 10-hydroxycarbazepine), and zopiclone (with metabolite $\mathrm{N}$-desmethylzopiclone). A reason for this can be the longer elimination half-life of these metabolites compared with their parent pharmaceuticals [112,113]. For clonazepam and flunitrazepam, the stronger basicity of the amino-metabolites can account for the higher incorporation of the metabolites [41]. Despite this, MD ratios below 1 were also reported for these pharmaceuticals, indicating that the MD ratios were also highly affected by other variables, including interindividual differences.

\subsection{Factors Affecting the MD Ratios in Hair}

\subsubsection{Inter- and Intraindividual Differences}

Interindividual differences may arise from differences in drug metabolism, hair color, cosmetic hair treatment, hair growth rate, the content of melanin in hair, sex, age, ethnicity, etc. Several studies have investigated the influence of interindividual differences on the MD ratio in hair. For instance, Papaseit et al. [92] found fluctuations in plasma concentrations after administering the same atomoxetine dose to different individuals and attributed this to interindividual differences in metabolism, including those due to the genetic polymorphism of CYP2D6, resulting in poor and extensive metabolizers. This can explain the differences in the reported $\mathrm{MD}$ ratios in hair ranging from 0.26 to 2.3 for atomoxetine and its metabolite 4-hydroxyatomoxetine $(n=6$, Table 1$)$. Other studies have likewise investigated the 
differences in metabolism due to the genetic polymorphisms of CYP2D6 and their influence on the MD ratios $[85,86,88]$. Johansen et al. [85] revealed a decreasing trend in the $O$ desmetyltramadol to tramadol ratio between extensive metabolizers of CYP2D6 (mean ratio: $0.30, n=8$ ), intermediate metabolizers of CYP2D6 (mean ratio: $0.21, n=8$ ), and the poor metabolizer of CYP2D6 (mean ratio: $0.072, n=1$ ), but an increasing trend of the $\mathrm{N}$-desmetyltramadol to tramadol ratio in the same phenotype groups (mean ratio: 0.27 , 0.34 , and 1.5, respectively). The same trends were observed by Yu et al. [86], and this is a reasonable explanation for the observed variations in the reported ratios. The MD ratios of tramadol and its metabolites were also reported in the same ranges from other studies but without considering drug metabolism $[20,28,87,91]$. Furthermore, the incorporation of tramadol from sweat and the wash-out effect should also be considered when evaluating MD ratios. Krumbiegel et al. [27] presented drug incorporation via sweat as a justification for the observed variations in MD ratios. They found that differences in the intensity of sweating as well as possible higher drug incorporation via sweat after methadone ingestion due to heavy perspiration to be plausible reasons for the variations in MD ratios. Increased perspiration is a common side effect of both methadone, tramadol, and many antipsychotics and antidepressants, and in such cases, concomitant drug intake may influence the ratios. In addition, the concomitant consumption of psychotropic medication metabolized by the same isoenzymes affects the drug metabolism and thus the MD ratio [92]. Musshoff et al. [89] reported strong inhibition of the enzyme CYP2B6 by selective serotonin reuptake inhibitors. CYP2B6 metabolizes ketamine to its metabolite norketamine, and concomitant drug intake may be a reason for varying MD ratios of ketamine and norketamine, which were reported from 0.010 to 1.3 in hair $(n=654$, see Table 1$)$.

For most of the MD ratios reported in the present review, the segment in which the MD ratio was found was not taken into account. Klys et al. [26] reported decreasing MD ratios of clomipramine and its metabolite $\mathrm{N}$-desmethylclomipramine in the segments situated closer to the hair bulb, indicating higher levels of metabolite in the segments closest to the scalp (proximal segments) and thus intraindividual variations in the MD ratio. This explains why MD ratios both below and above 1 have been reported. Similar findings were observed for methadone, quetiapine, risperidone, and tramadol $[27,31,39,85]$. Other studies revealed only small variations in the MD ratios in all hair segments, from the proximal end to the distal end, for patients with a presumed long-term intake of either aripiprazole, chlorprothixene, or clozapine $[31,34,35,63]$. Similar results were reported for a single dose study of zopiclone in $0.5 \mathrm{~cm}$ segments up to four months after ingestion by Hansen et al. [63]. Total intraindividual variations in MD ratios of less than $20 \%$ of zopiclone and aripiprazole have been reported $[34,63]$. However, these studies did not elaborate further on factors such as hair pigmentation or cosmetic and physical hair treatment, which can influence the amount of pharmaceuticals or metabolites measured in hair.

\subsubsection{Hair Characteristic}

Kronstrand et al. [36] found significantly different mean MD ratios of clozapine and its metabolite $N$-desmethylclozapine in pigmented $(1.2, n=12)$ and non-pigmented $(0.93$, $n=11$ ) hair, indicating better incorporation of the metabolites into pigmented hair (See Table 1). Higher nordiazepam than diazepam concentrations was also reported in black hair after a single dose of diazepam $(n=8)$ [47]. In contrast, Wang et al. [31] presented MD ratios of aripiprazole and its metabolite dehydroaripiprazole in black hair, which were one-tenth of the other reported MD ratios, indicating better incorporation of the parent pharmaceutical in black hair (pigmented hair) [34]. Licata et al. [20] reported higher levels of the metabolite nortriptyline compared to amitriptyline for patients with hair that is gray or subjected to cosmetic treatment, which can explain the MD ratios greater than 1 mentioned earlier. Likewise, olanzapine concentrations were found to be low in gray hair [37]. These observations reaffirm the influence of hair characteristics on the MD ratios of some pharmaceuticals. Fernández et al. [114] determined the influence of hair color and melanin content on hair analysis by measuring the antipsychotic drugs 
chlorpromazine, haloperidol, olanzapine, quetiapine, and risperidone (+ metabolites) in the melanin and protein fractions of collected hair samples. They found that hair melanin had a higher affinity for the five antipsychotic drugs compared to the protein fraction but that the results were influenced more by the effects of biochemical individuality and less by hair color. Unfortunately, in forensic investigations, information on these variables and on the actual intake are often limited, which may complicate the interpretation of the hair analysis results.

\subsubsection{Drug Intake}

MD ratios may be a helpful tool to differentiate between actual intake and external contamination. Madry et al. [88] investigated MD ratios in individuals with a passive exposure working at a tramadol production company. They found that the O-desmethyltramadol to tramadol ratios in tramadol users were significantly higher than in subjects with passive exposure. They suggested an MD ratio of tramadol and its metabolite $O$-desmethyltramadol of 0.021 and an MD ratio of tramadol and N-desmethyltramadol of 0.037 to be used to distinguish drug ingestion from external contamination. This distinction is a major problem especially for drugs of abuse, whereas contamination with pharmaceuticals to a greater extent is a problem when distinguishing a single ingestion in terms of intoxication from long-term intake. Such differentiations are only possible with segmental hair analysis. In the present review, no trends were found indicating lower or higher MD ratios in single-dose compared with long-term use. However, only a few of the included studies were controlled single-dose or long-term use studies. For pharmaceuticals with a short elimination half-life, MD ratios above 1 might be expected after single dose intake, whereas MD ratios after long-term use may rather be dependent upon other factors such as compound properties due to steady state plasma concentrations. Despite this, Xiang et al. [106] reported very high ketamine concentrations and low norketamine concentrations in the hair of drug abusers after occasional use of ketamine, indicating the great influence on MD by other factors. In many of the studies, it was not clear whether the pharmaceuticals were used or abused, and in general it is difficult to distinguish therapeutic use from abuse. No clear differences were observed in the MD ratios for drug use cases compared with drug abuse cases. In addition, Papaseit et al. [92] found no linear relationship between the administered daily dose, daily dose per kg weight, and the MD ratio of atomoxetine and its metabolite 4-hydroxyatomoxetine in hair segments from any of the included individuals $(n=6)$.

Another factor to be considered is metabolites serving as prescription drugs themselves, including 9-OH-risperidone, lorazepam, nordiazepam, norfluoxetine, nortriptyline, oxazepam, oxymorphone, and temazepam. In addition, some pharmaceuticals, especially benzodiazepines, are formed from more than one parent pharmaceutical. In such cases, the MD ratios may be artificially elevated, which causes wide-range MD ratios and complicates the interpretation and comparison of MD ratios both within and between studies.

\subsubsection{Pre-Analytical Workflow and Analytics}

Skopp et al. [115] reported a potential underestimation of buprenorphine and norbuprenorphine concentrations in hair and reversed concentration ratios if these compounds were recovered by acidic procedures. This indicates that the sample preparation procedure may also be a cause for the different MD ratios reported. Kintz [6] described the possibilities of postmortem incorporation of drugs into hair through blood, sweat, etc., demonstrating that determining accurate MD ratios also critically depends on effective washing procedures for the sufficient removal of external contamination [6,116]. Postmortem incorporation via sweat is critical because sweat may contain both the parent compound and the metabolite, complicating the interpretation of the determined concentrations in hair and MD ratios [19]. Other variables in the sample preparation process, including differences in sample collection, segmentation, and preparation of hair samples (wash procedure and the powering or cutting hair before extraction), may influence the $\mathrm{MD}$ ratio as well. 
Table 1 highlights the frequent use of LC-MS as well as GC-MS methods for hair analysis. A benefit of using GC-MS is more robustness against the matrix effect. The matrix effects may suppress or enhance the analyte signal during analysis, leading to inaccurate analysis results. LC-MS methods are more highly influenced by the matrix effect, and in such cases, the choice of internal standard is highly important to obtaining accurate analysis results. Higher accuracies are associated with coeluting isotope-labeled internal analogs, as they allow for better correction. More than 50\% of the MD ratios were reported from studies that did not involve the use of isotope-labeled internal analogs for either the pharmaceutical or the metabolite, and this too can explain the wide-ranging MD ratios.

Finally, better comparability among different methods and laboratories can be established from external proficiency testing, which unfortunately is still limited for many pharmaceuticals. Thus, we encourage researchers in the field of forensic hair analysis to report MD ratios in hair in order to obtain more reference values and to better understand the factors influencing the ratios. The overview of MD ratios in this review provides guidance for evaluating MD ratios in the future.

\subsection{Limitations}

This review is based on MD ratios reported or calculated from other studies; therefore, the information available was limited. In addition, inconsistency in the way the ratios were reported, varieties in hair and segment length, and the number of subjects in the different studies complicated the comparison of the MD ratios. Due to the inconsistency in the way the MD ratios were reported, this review showed MD ratio ranges based on the minimum and maximum values together with median intervals, including the lowest reported median and the highest median, if available. MD ratio ranges found only from minimum and maximum values contribute to the wide-ranging MD ratio ranges observed. Therefore, MD ratios reported as 10-90 percentile ranges would be preferred as these exclude the extreme values and thus improve the range for interpretation.

As seen from Table 1, MD ratios have been reported for different types of cases, including deceased individuals, individuals in treatment, and individuals with drug abuse. In many of the postmortem cases, it was not clear whether the intake of the particular pharmaceutical led to intoxication and was the cause of death or if the pharmaceutical were taken regularly. Such differentiations are only possible with segmental analysis, which has only been occasionally published in the studies. These limitations minimize the possibilities to further interpret MD ratios across different case types.

Furthermore, it was not possible in all cases to identify whether metabolites served as pharmaceuticals themselves or were metabolized from multiple pharmaceuticals, leading to discrepancies in the MD ratios. The wide range of reported MD ratios of the pharmaceuticals highlighted in the present review may limit the interpretation of MD ratios, and, therefore, MD ratios need to be reported in a more consistent way, and need to be studied further.

\section{Conclusions}

The present review provides a comprehensive overview of the metabolite-to-drug (MD) ratios of pharmaceuticals in hair, a topic that is very limited in the literature. MD ratios of 41 pharmaceuticals from 94 studies were reported. The most frequently reported were MD ratios below 1, indicating that the parent pharmaceutical concentration in hair exceeded metabolite concentration in most cases. In addition, the review highlights that MD ratios of a majority of the pharmaceuticals were wide-ranging. Among others, intra- and interindividual differences and compound properties were variables that could possibly have contributed to this. This overview of MD ratios and medians can provide helpful guidance for the comparison and evaluation of MD ratios of pharmaceuticals in hair reported in future research. 
Author Contributions: All authors contributed to this systematic review. Conceptualization, K.R., K.L. and S.S.J.; methodology, K.R., K.L. and S.S.J.; investigation, K.R.; data curation, K.R., K.L. and S.S.J.; writing—original draft preparation, K.R.; writing—review and editing, K.R., K.L. and S.S.J.; visualization, K.R., K.L. and S.S.J.; supervision, K.L. and S.S.J.; project administration, K.R. All authors have read and agreed to the published version of the manuscript.

Funding: This research received no external funding.

Acknowledgments: Not applicable.

Conflicts of Interest: The authors declare no conflict of interest.

\section{References}

1. Kintz, P. Value of hair analysis in postmortem toxicology. Forensic Sci. Int. 2004, 142, 127-134. [CrossRef]

2. Hansen, S.H.; Pedersen-Bjergaard, S.; Reubsaet, L.; Grønhaug Halvorsen, T.; Gjelstad, A.; Jørgensen, M.; Kall, M.A. Bioanalysis of Pharmaceuticals: Sample Preparation, Separation Techniques and Mass Spectrometry, 1st ed.; Wiley: Hoboken, NJ, USA, 2015; ISBN 9781118716830.

3. Pragst, F.; Balikova, M.A. State of the art in hair analysis for detection of drug and alcohol abuse. Clin. Chim. Acta 2006, 370, 17-49. [CrossRef]

4. Wennig, R. Potential problems with the interpretation of hair analysis results. Forensic Sci. Int. 2000, 107, 5-12. [CrossRef]

5. Cooper, G.A.A.; Kronstrand, R.; Kintz, P. Society of hair testing guidelines for drug testing in hair. Forensic Sci. Int. 2012, 218, 20-24. [CrossRef]

6. Kintz, P. Segmental hair analysis can demonstrate external contamination in postmortem cases. Forensic Sci. Int. 2012, 215, 73-76. [CrossRef]

7. Society of Hair Testing. Statement of the society of hair testing concerning the examination of drugs in human hair. Forensic Sci. Int. 1997, 84, 3-6.

8. Nielsen, M.K.K.; Johansen, S.S.; Linnet, K. Evaluation of poly-drug use in methadone-related fatalities using segmental hair analysis. Forensic Sci. Int. 2015, 248, 134-139. [CrossRef]

9. Zhuo, Y.; Xiang, P.; Wu, J.; Wang, X. Segmental hair analysis for flunitrazepam and 7-aminoflunitrazepam in users: A comparison to existing literature. Forensic Sci. Res. 2020, 1-9. [CrossRef]

10. Morris-Kukoski, C.L.; Montgomery, M.A.; Hammer, R.L. Analysis of extensively washed hair from cocaine users and drug chemists to establishnew reporting criteria. J. Anal. Toxicol. 2014, 38, 628-636. [CrossRef]

11. Han, E.; Park, Y.; Yang, W.; Lee, J.; Lee, S.; Kim, E.; Lim, M.; Chung, H. The study of metabolite-to-parent drug ratios of methamphetamine and methylenedioxymethamphetamine in hair. Forensic Sci. Int. 2006, 161, 124-129. [CrossRef]

12. Franz, T.; Scheufler, F.; Stein, K.; Uhl, M.; Dame, T.; Schwarz, G.; Sachs, H.; Skopp, G.; Musshoff, F. Determination of hydroxy metabolites of cocaine from hair samples and comparison with street cocaine samples. Forensic Sci. Int. 2018, 288, 223-226. [CrossRef]

13. Ropero-Miller, J.D.; Huestis, M.A.; Stout, P.R. Cocaine analytes in human hair: Evaluation of concentration ratios in different cocaine sources, drug-user populations and surface-contaminated specimens. J. Anal. Toxicol. 2012, 36, 390-398. [CrossRef]

14. Methling, M.; Krumbiegel, F.; Hartwig, S. Hair analysis of antidepressants and antipsychotics-Overview of quantitative data. Drug Test. Anal. 2020, 12, 659-676. [CrossRef]

15. Vogliardi, S.; Favretto, D.; Tucci, M.; Stocchero, G.; Ferrara, S.D. Simultaneous LC-HRMS determination of 28 benzodiazepines and metabolites in hair. Anal. Bioanal. Chem. 2011, 400, 51-67. [CrossRef]

16. Moher, D.; Liberati, A.; Tetzlaff, J.; Altman, D.G.; Group, T.P. Preferred reporting items for systematic reviews and meta-analyses: The PRISMA Statement. PLoS Med. 2009, 6, e1000097. [CrossRef]

17. Liberati, A.; Altman, D.G.; Tetzlaff, J.; Mulrow, C.; Gøtzsche, P.C.; Ioannidis, J.P.A.; Clarke, M.; Devereaux, P.J.; Kleijnen, J.; Moher, D. The PRISMA statement for reporting systematic reviews and meta-analyses of studies that evaluate health care interventions: Explanation and elaboration. BMJ 2009, 339, b2700. [CrossRef]

18. Methling, M.; Krumbiegel, F.; Alameri, A.; Hartwig, S.; Parr, M.; Tsokos, M. Concentrations of antidepressants, antipsychotics, and benzodiazepines in hair samples from postmortem cases. SN Compr. Clin. Med. 2020, 2, 284-300. [CrossRef]

19. Fernández, M.D.M.R.; Wille, S.M.R.R.; Hill, V.; Samyn, N.; Fernádez, M.d.M.; Wille, S.M.R.R.; Hill, V.; Samyn, N. Determination of antidepressants in hair via UHPLC-MS/MS as a complementary informative tool for clinical and forensic toxicological assessments. Ther. Drug Monit. 2016, 38, 751-760. [CrossRef]

20. Licata, M.; Rustichelli, C.; Palazzoli, F.; Ferrari, A.; Baraldi, C.; Vandelli, D.; Verri, P.; Marchesi, F.; Silingardi, E. Hair testing in clinical setting: Simultaneous determination of 50 psychoactive drugs and metabolites in headache patients by LC tandem MS. J. Pharm. Biomed. Anal. 2016, 126, 14-25. [CrossRef]

21. Fisichella, M.; Morini, L.; Sempio, C.; Groppi, A. Validation of a multi-analyte LC-MS/MS method for screening and quantification of 87 psychoactive drugs and their metabolites in hair. Anal. Bioanal. Chem. 2014, 406, 3497-3506. [CrossRef]

22. Nielsen, M.K.K.; Johansen, S.S.; Dalsgaard, P.W.; Linnet, K. Simultaneous screening and quantification of 52 common pharmaceuticals and drugs of abuse in hair using UPLC-TOF-MS. Forensic Sci. Int. 2010, 196, 85-92. [CrossRef] 
23. Pragst, F.; Rothe, M.; Hunger, J.; Thor, S. Structural and concentration effects on the deposition of tricyclic antidepressants in human hair. Forensic Sci. Int. 1997, 84, 225-236. [CrossRef]

24. Ishiyama, I.; Nagai, T.; Toshida, S. Detection of basic drugs (methamphetamine, antidepressants, and nicotine) from human hair. J. Forensic Sci. 1983, 28, 380-385. [CrossRef]

25. Wille, S.M.R.; De Letter, E.A.; Piette, M.H.A.; Van Overschelde, L.K.; Van Peteghem, C.H.; Lambert, W.E. Determination of antidepressants in human postmortem blood, brain tissue, and hair using gas chromatography-mass spectrometry. Int. J. Legal Med. 2009, 123, 451-458. [CrossRef]

26. Kłys, M.; Scisłowski, M.; Rojek, S.; Kołodziej, J. A fatal clomipramine intoxication case of a chronic alcoholic patient: Application of postmortem hair analysis method of clomipramine and ethyl glucuronide using LC/APCI/MS. Leg. Med. 2005, 7, 319-325. [CrossRef]

27. Krumbiegel, F.; Hastedt, M.; Westendorf, L.; Niebel, A.; Methling, M.; Parr, M.K.; Tsokos, M. The use of nails as an alternative matrix for the long-term detection of previous drug intake: Validation of sensitive UHPLC-MS/MS methods for the quantification of 76 substances and comparison of analytical results for drugs in nail and hair samples. Forensic Sci. Med. Pathol. 2016, 12, 416-434. [CrossRef]

28. Wang, X.; Johansen, S.S.; Nielsen, M.K.K.; Linnet, K. Hair analysis in toxicological investigation of drug-facilitated crimes in Denmark over a 8-year period. Forensic Sci. Int. 2018, 285, e1-e12. [CrossRef]

29. Couper, F.; Mclntyre, I.; Drummer, O. Detection of antidepressant and antipsychotic drugs in postmortem human scalp hair. J. Forensic Sci. 1995, 40, 87-90. [CrossRef]

30. Kintz, P.; Raul, S.; Ameline, A. Evidence of repeated mirtazapine poisoning in children by hair analysis. J. Forensic Sci. 2021, 66, 1165-1170. [CrossRef]

31. Wang, X.; Zhuo, Y.; Tang, X.; Qiang, H.; Liu, W.; Wu, H.; Xiang, P.; Duan, G.; Shen, M. Segmental analysis of antidepressant and antipsychotic drugs in the hair of schizophrenic patients. Drug Test. Anal. 2019, 12, 1-13. [CrossRef]

32. Marchei, E.; Palmi, I.; Pichini, S.; Pacifici, R.; Anton Airaldi, I.-R.; Costa Orvay, J.A.; Garcia Serra, J.; Bonet Serra, B.; Garcia-Algar, O. Segmental hair testing to disclose chronic exposure to psychoactive drugs. Adicciones 2016, 28, 158-162. [CrossRef]

33. Pichini, S.; Cortes, L.; Marchei, E.; Solimini, R.; Pacifici, R.; Gomez-Roig, M.D.; García-Algar, O. Ultra-high-pressure liquid chromatography tandem mass spectrometry determination of antidepressant and anxiolytic drugs in neonatal meconium and maternal hair. J. Pharm. Biomed. Anal. 2016, 118, 9-16. [CrossRef]

34. Rygaard, K.; Linnet, K.; Banner, J.; Johansen, S.S. Concentrations of aripiprazole and dehydroaripiprazole in hair segments from deceased individuals with mental disorders. Forensic Sci. Int. 2020, 317, 110523. [CrossRef]

35. Günther, K.N.; Johansen, S.S.; Wicktor, P.; Banner, J.; Linnet, K. Segmental analysis of chlorprothixene and desmethylchlorprothixene in postmortem hair. J. Anal. Toxicol. 2018, 42, 642-649. [CrossRef]

36. Kronstrand, R.; Roman, M.; Hedman, M.; Ahlner, J.; Dizdar, N. Dose-hair concentration relationship and pigmentation effects in patients on low-dose clozapine. Forensic Sci. Med. Pathol. 2007, 3, 107-114. [CrossRef]

37. Günther, K.N.; Banner, J.; Linnet, K.; Johansen, S.S. Segmental hair analysis of olanzapine and N-desmethyl-olanzapine in postmortem hair from mentally ill patients by LC-MS/MS. J. Pharm. Biomed. Anal. 2020, 190, 113510. [CrossRef]

38. Schneider, S.; Sibille, E.; Yegles, M.; Neels, H.; Wennig, R.; Mühe, A. Time resolved analysis of risperidone and 9-hydroxyrisperidone in hair using LC/MS-MS. J. Chromatogr. B Anal. Technol. Biomed. Life Sci. 2009, 877, 2589-2592. [CrossRef]

39. Binz, T.M.; Yegles, M.; Schneider, S.; Neels, H.; Crunelle, C.L. Time resolved analysis of quetiapine and 7-OH-quetiapine in hair using LC/MS-MS. Forensic Sci. Int. 2014, 242, 200-203. [CrossRef]

40. Wang, X.; Johansen, S.S.; Nielsen, M.K.K.; Linnet, K. Targeted analysis of 116 drugs in hair by UHPLC-MS/MS and its application to forensic cases. Drug Test. Anal. 2017, 9, 1137-1151. [CrossRef]

41. Madry, M.M.; Kraemer, T.; Baumgartner, M.R. Large scale consumption monitoring of benzodiazepines and z-drugs by hair analysis. J. Pharm. Biomed. Anal. 2020, 183, 113151. [CrossRef]

42. Shin, Y.; Kong, T.Y.; Cheong, J.C.; Kim, J.Y.; Lee, J.; Lee, H.S. Simultaneous determination of 75 abuse drugs including amphetamines, benzodiazepines, cocaine, opioids, piperazines, zolpidem and metabolites in human hair samples using liquid chromatography-tandem mass spectrometry. Biomed. Chromatogr. 2019, 33, e4600. [CrossRef]

43. Chéze, M.; Duffort, G.; Deveaux, M.; Pépin, G. Hair analysis by liquid chromatography-Tandem mass spectrometry in toxicological investigation of drug-facilitated crimes: Report of 128 cases over the period June 2003-May 2004 in metropolitan Paris. Forensic Sci. Int. 2005, 153, 3-10. [CrossRef]

44. Negrusz, A.; Moore, C.M.; Kern, J.L.; Philip, G.; Strong, M.J.; Levy, N.A. Quantitation of clonazepam and its major metabolite 7-aminoclonazepam in hair. J. Anal. Toxicol. 2000, 24, 614-620. [CrossRef]

45. Lendoiro, E.; de Castro, A.; Jiménez-Morigosa, C.; Gomez-Fraguela, X.A.; López-Rivadulla, M.; Cruz, A. Usefulness of hair analysis and psychological tests for identification of alcohol and drugs of abuse consumption in driving license regranting. Forensic Sci. Int. 2018, 286, 239-244. [CrossRef]

46. Morini, L.; Pozzi, F.; Groppi, A. Stability of benzodiazepines in hair after prolonged exposure to chlorinated water. Forensic Sci. Int. 2017, 278, 217-220. [CrossRef]

47. Wang, X.; Johansen, S.S.; Zhang, Y.; Jia, J.; Rao, Y.; Jiang, F.; Linnet, K. Deposition of diazepam and its metabolites in hair following a single dose of diazepam. Int. J. Legal Med. 2017, 131, 131-141. [CrossRef] 
48. Kim, J.; Yum, H.; Jang, M.; Shin, I.; Yang, W.; Baeck, S.; Suh, J.H.; Lee, S.; Han, S.B. A comprehensive and sensitive method for hair analysis in drug-facilitated crimes and incorporation of zolazepam and tiletamine into hair after a single exposure. Anal. Bioanal. Chem. 2016, 408, 251-263. [CrossRef]

49. Maublanc, J.; Dulaurent, S.; Morichon, J.; King, L. Identification and quantification of 35 psychotropic drugs and metabolites in hair by LC-MS/MS: Application in forensic toxicology. Int. J. Leg. Med. 2015, 129, 259-268. [CrossRef]

50. Lendoiro, E.; Quintela, Ó.; de Castro, A.; Cruz, A.; López-Rivadulla, M.; Concheiro, M. Target screening and confirmation of 35 licit and illicit drugs and metabolites in hair by LC-MSMS. Forensic Sci. Int. 2012, 217, 207-215. [CrossRef]

51. Favretto, D.; Vogliardi, S.; Stocchero, G.; Nalesso, A.; Tucci, M.; Ferrara, S.D. High performance liquid chromatographyhigh resolution mass spectrometry and micropulverized extraction for the quantification of amphetamines, cocaine, opioids, benzodiazepines, antidepressants and hallucinogens in $2.5 \mathrm{mg}$ hair samples. J. Chromatogr. A 2011, 1218, 6583-6595. [CrossRef]

52. Kim, J.; Lee, S.; In, S.; Choi, H.; Chung, H. Validation of a simultaneous analytical method for the detection of 27 benzodiazepines and metabolites and zolpidem in hair using LC-MS/MS and its application to human and rat hair. J. Chromatogr. B 2011, 879, 878-886. [CrossRef]

53. Lee, S.; Han, E.; In, S.; Choi, H.; Chung, H.; Chung, K.H. Determination of illegally abused sedative-hypnotics in hair samples from drug offenders. J. Anal. Toxicol. 2011, 35, 312-315. [CrossRef] [PubMed]

54. Miller, E.I.; Wylie, F.M.; Oliver, J.S. Simultaneous detection and quantification of amphetamines, diazepam and its metabolites, cocaine and its metabolites, and opiates in hair by LC-ESI-MS-MS using a single extraction method. J. Anal. Toxicol. 2008, 32, 457-469. [CrossRef] [PubMed]

55. Ariffin, M.M.; Miller, E.I.; Cormack, P.A.G.; Anderson, R.A. Molecularly imprinted solid-phase extraction of diazepam and its metabolites from hair samples. Anal. Chem. 2007, 79, 256-262. [CrossRef] [PubMed]

56. Yegles, M.; Mersch, F.; Wennig, R. Detection of benzodiazepines and other psychotropic drugs in human hair by GC/MS. Forensic Sci. Int. 1997, 84, 211-218. [CrossRef]

57. Negrusz, A.; Moore, C.; Deitermann, D.; Lewis, D.; Kaleciak, K.; Kronstrand, R.; Feeley, B.; Niedbala, R.S. Highly sensitive micro-plate enzyme immunoassay screening and NCI-GC-MS confirmation of flunitrazepam and its major metabolite 7-aminoflunitrazepam in hair. J. Anal. Toxicol. 1999, 23, 429-435. [CrossRef]

58. Cirimele, V.; Kintz, P.; Staub, C.; Mangin, P. Testing human hair for flunitrazepam and 7-amino-flunitrazepam by GC/MS-NCI. Forensic Sci. Int. 1997, 84, 189-200. [CrossRef]

59. Cirimele, V.; Kintz, P.; Mangin, P. Determination of chronic flunitrazepam abuse by hair analysis using GC-MS-NCi. J. Anal. Toxicol. 1996, 20, 20-22. [CrossRef]

60. Wiart, J.; Hakim, F.; Andry, A.; Eiden, C.; Drevin, G.; Lelièvre, B.; Rougé-maillart, C.; Decourcelle, M.; Allorge, D. Pitfalls of toxicological investigations in hair, bones and nails in extensively decomposed bodies: Illustration with two cases. Int. J. Leg. Med. 2020, 134, 1339-1344. [CrossRef]

61. Kintz, P.; Cirimele, V.; Vayssette, F.; Mangin, P. Hair analysis for nordiazepam and oxazepam by gas chromatography-negative-ion chemical ionization mass spectrometry. J. Chromatogr. B Biomed. Sci. Appl. 1996, 677, 241-244. [CrossRef]

62. Toyo, T.; Kanbori, M.; Kumaki, Y.; Nakahara, Y. Determination of triazolam involving its hydroxy metabolites in hair shaft and hair root by reversed-phase liquid chromatography with electrospray ionization mass spectrometry and application to human hair analysis. Anal. Biochem. 2001, 179, 172-179. [CrossRef]

63. Hansen, S.L.; Johansen, S.S.; Nielsen, M.K.K.; Nilsson, G.; Kronstrand, R. Distribution of zopiclone and main metabolites in hair following a single dose. Forensic Sci. Int. 2020, 306, 110074. [CrossRef]

64. Belivanis, S.; Tzatzarakis, M.N.; Vakonaki, E.; Kovatsi, L.; Mantsi, M.; Alegakis, A.; Kavvalakis, M.P.; Vynias, D.; Tsatsakis, A.M. Buprenorphine and nor-buprenorphine levels in head hair samples from former heroin users under Suboxone ${ }^{\circledR}$ treatment. Drug Test. Anal. 2014, 6 (Suppl. 1), 93-100. [CrossRef]

65. Vincent, F.; Bessard, J.; Vacheron, J.; Mallaret, M.; Bessard, G. Determination of buprenorphine and norbuprenorphine in urine and hair by gas chromatography-mass spectrometry. J. Anal. Toxicol. 1999, 23, 270-279. [CrossRef] [PubMed]

66. Kintz, P.; Cirimele, V.; Edel, Y.; Jamey, C. Hair analysis for buprenorphine and its dealkylated metabolite by RIA and confirmation by LC/ECD. J. Forensic Sci. 1994, 39, 1497-1503. [CrossRef]

67. Kim, J.; Ji, D.; Kang, S.; Park, M.; Yang, W.; Kim, E.; Choi, H.; Lee, S. Simultaneous determination of 18 abused opioids and metabolites in human hair using LC-MS/MS and illegal opioids abuse proven by hair analysis. J. Pharm. Biomed. Anal. 2014, 89, 99-105. [CrossRef]

68. Kim, J.Y.; Suh, S.I.; Paeng, K.; In, M.K. Determination of dextromethorphan and its metabolite dextrorphan in human hair by gas chromatography-mass spectrometry. Chromatographia 2004, 60, 703-707. [CrossRef]

69. Salomone, A.; Bigiarini, R.; Palamar, J.J.; McKnight, C.; Vinsick, L.; Amante, E.; Di Corcia, D.; Vincenti, M. Toward the interpretation of positive testing for fentanyl and its analogs in real hair samples: Preliminary considerations. J. Anal. Toxicol. 2020, 44, 362-369. [CrossRef] [PubMed]

70. Palamar, J.J.; Salomone, A.; Bigiarini, R.; Vincenti, M.; Acosta, P.; Tofighi, B.; Palamar, J.J.; Salomone, A.; Bigiarini, R.; Vincenti, M. Testing hair for fentanyl exposure: A method to inform harm reduction behavior among individuals who use heroin. Am. J. Drug Alcohol Abuse 2019, 45, 90-96. [CrossRef] [PubMed]

71. Min, S.; Ping, X.; Baohua, S. Detection of meperidine and its metabolites in the hair of meperidine addicts. Forensic Sci. Int. 1999, 103, 159-171. [CrossRef] 
72. Rosado, T.; Gallardo, E. Microextraction by packed sorbent as a novel strategy for sample clean-up in the determination of methadone and EDDP in hair. J. Anal. Toxicol. 2020, 44, 840-850. [CrossRef]

73. Cappelle, D.; De Keukeleire, S.; Neels, H.; Been, F.; De Doncker, M.; Dom, G.; Crunelle, C.L.; Covaci, A.; van Nuijs, A.L.N. Keratinous matrices for the assessment of drugs of abuse consumption: A correlation study between hair and nails. Drug Test. Anal. 2018, 10, 1110-1118. [CrossRef]

74. Cappelle, D.; De Doncker, M.; Gys, C.; Krysiak, K.; De Keukeleire, S.; Maho, W.; Crunelle, C.L.; Dom, G.; Covaci, A.; van Nuijs, A.L.N.; et al. A straightforward, validated liquid chromatography coupled to tandem mass spectrometry method for the simultaneous detection of nine drugs of abuse and their metabolites in hair and nails. Anal. Chim. Acta 2017, 960, 101-109. [CrossRef] [PubMed]

75. Kintz, P.; Farrugia, A.; Ameline, A.; Eibel, A.; Raul, J. High risk of misinterpreting hair analysis results for children tested for methadone. Forensic Sci. Int. 2017, 280, 176-180. [CrossRef]

76. Joya, X.; Marchei, E.; Salat-batlle, J.; García-Algar, O. Drugs of abuse in maternal hair and paired neonatal meconium: An objective assessment of foetal exposure to gestational consumption. Drug Test. Anal. 2016, 8, 864-868. [CrossRef] [PubMed]

77. Koster, R.A.; Alffenaar, J.-W.C.; Greijdanus, B.; VanDernagel, J.E.L.; Uges, D.R.A. Fast and highly selective LC-MS/MS screening for THC and 16 other abused drugs and metabolites in human hair to monitor patients for drug abuse. Ther. Drug Monit. 2014, 36, 234-243. [CrossRef] [PubMed]

78. Tournel, G.; Pollard, J.; Humbert, L.; Wiart, J.-F.; Héouin, V.; Allorge, D. Use of hair testing to determine methadone exposure in pediatric deaths. J. Forensic Sci. 2014, 59, 1436-1440. [CrossRef] [PubMed]

79. Favretto, D.; Tucci, M.; Monaldi, A.; Ferrara, S.D.; Miolo, G.; Davide, S.; Miolo, G. A study on photodegradation of methadone, EDDP, and other drugs of abuse in hair exposed to controlled UVB radiation. Drug Test. Anal. 2014, 6, 78-84. [CrossRef]

80. Fucci, N.; De Giovanni, N. Methadone in hair and sweat from patients in long-term maintenance therapy. Ther. Drug Monit. 2007, 29, 452-454. [CrossRef]

81. Lucas, A.C.S.; Bermejo, A.M.; Tabernero, M.J.; Fernandez, P. Use of solid-phase microextraction (SPME) for the determination of methadone and EDDP in human hair by GC-MS. Forensic Sci. Int. 2000, 107, 225-232. [CrossRef]

82. Sporkert, F.; Pragst, F. Determination of methadone and its metabolites EDDP and EMDP in human hair by headspace solid-phase microextraction and gas chromatography-mass spectrometry. J. Chromatogr. B Biomed. Sci. Appl. 2000, 746, 255-264. [CrossRef]

83. Moeller, M.; Fey, P.; Wennig, R. Simultaneous determination of drugs of abuse in human hair by GC/MS and its application to a methadone treatment program. Forensic Sci. Int. 1993, 63, 185-206. [CrossRef]

84. Reisfield, G.M.; Jones, J.T. the disposition of oxycodone and metabolite in human hair. J. Anal. Toxicol. 2015, 39, 746-750. [CrossRef]

85. Johansen, S.S.; Le Dang, L.T.V.; Nielsen, M.K.K.; Haage, P.; Kugelberg, F.C.; Kronstrand, R. Temporal patterns of tramadol in hair after a single dose. Forensic Sci. Int. 2020, 316, 110546. [CrossRef]

86. Yu, H.; Choi, M.; Jang, J.-H.; Park, B.; Seo, Y.H.; Jeong, C.-H.; Bae, J.-W.; Lee, S. Development of a column-switching LC-MS/MS method of tramadol and its metabolites in hair and application to a pharmacogenetic study. Arch. Pharm. Res. 2018, 41, 554-563. [CrossRef] [PubMed]

87. Verri, P.; Rustichelli, C.; Palazzoli, F.; Vandelli, D.; Marchesi, F.; Ferrari, A.; Licata, M. Tramadol chronic abuse: An evidence from hair analysis by LC tandem MS. J. Pharm. Biomed. Anal. 2015, 102, 450-458. [CrossRef] [PubMed]

88. Madry, M.M.; Rust, K.Y.; Guglielmello, R.; Baumgartner, M.R.; Kraemer, T. Metabolite to parent drug concentration ratios in hair for the differentiation of tramadol intake from external contamination and passive exposure. Forensic Sci. Int. 2012, 223, 330-334. [CrossRef] [PubMed]

89. Musshoff, F.; Schwarz, G.; Sachs, H.; Skopp, G.; Franz, T. Concentration distribution of more than 100 drugs and metabolites in forensic hair samples. Int. J. Legal Med. 2020, 134, 989-995. [CrossRef]

90. Wang, X.; Johansen, S.S.; Nielsen, M.K.K.; Linnet, K. Segmental hair analysis-Interpretation of the time of drug intake in two patients undergoing drug treatment. J. Forensic Sci. 2019, 64, 950-955. [CrossRef] [PubMed]

91. Pinho, S.; Oliveira, A.; Costa, I.; Alexandra, C.; Carvalho, F.; Falcão, R. Simultaneous quantification of tramadol and Odesmethyltramadol in hair samples by gas chromatography-Electron impact/mass spectrometry. Biomed. Chromatogr. 2013, 23, 1003-1011. [CrossRef] [PubMed]

92. Papaseit, E.; Marchei, E.; Mortali, C.; Aznar, G.; Garcia-Algar, O.; Farrè, M.; Pacifici, R.; Pichini, S. Development and validation of a liquid chromatography-tandem mass spectrometry assay for hair analysis of atomoxetine and its metabolites: Application in clinical practice. Forensic Sci. Int. 2012, 218, 62-67. [CrossRef]

93. Kim, J.Y.; In, M.K.; Paeng, K.; Chung, B.C. Simultaneous determination of carisoprodol and meprobamate in human hair using solid-phase extraction and gas chromatography / mass spectrometry of the trimethylsilyl derivatives. Rapid Commun. Mass Spectrom. 2005, 19, 3056-3062. [CrossRef]

94. Zhuo, Y.; Wang, X.; Wu, J.; Zhang, S.; Deng, H.; Liu, W.; Wu, H.; Xiang, P.; Shen, M. Simultaneous quantitative determination of amphetamines, opiates, ketamine, cocaine and metabolites in human hair: Application to forensic cases of drug abuse. J. Forensic Sci. 2020, 65, 563-569. [CrossRef]

95. Miolo, G.; Tucci, M.; Id, L.M.; Stocchero, G.; Vogliardi, S.; Scrivano, S.; Montisci, M.; Favretto, D. A Study on photostability of amphetamines and ketamine in hair irradiated under artificial sunlight. Brain Sci. 2018, 8, 96. [CrossRef] 
96. Leung, K.W.; Wong, Z.C.F.; Ho, J.Y.M.; Yip, A.W.S.; Ng, J.S.C.; Ip, S.P.H.; Ng, W.Y.Y.; Ho, K.K.L.; Duan, R.; Zhu, K.Y.; et al. Determination of hair ketamine cut-off value from Hong Kong ketamine users by LC-MS/MS analysis. Forensic Sci. Int. 2016, 259, 53-58. [CrossRef]

97. Salomone, A.; Gerace, E.; Diana, P.; Romeo, M.; Malvaso, V.; Di Corcia, D.; Vincenti, M. Cut-off proposal for the detection of ketamine in hair. Forensic Sci. Int. 2015, 248, 119-123. [CrossRef] [PubMed]

98. Chang, Y.; Chao, M.; Chen, S.; Chen, C. A high-throughput method based on microwave-assisted extraction and liquid chromatography-tandem mass spectrometry for simultaneous analysis of amphetamines, ketamine, opiates, and their metabolites in hair. Anal. Bioanal. Chem. 2014, 406, 2445-2455. [CrossRef] [PubMed]

99. Favretto, D.; Vogliardi, S.; Stocchero, G.; Nalesso, A.; Tucci, M.; Terranova, C.; Davide, S. Determination of ketamine and norketamine in hair by micropulverized extraction and liquid chromatography-high resolution mass spectrometry. Forensic Sci. Int. 2013, 226, 88-93. [CrossRef] [PubMed]

100. Zhu, K.Y.; Leung, K.W.; Ting, A.K.L.; Wong, Z.C.F.; Fu, Q.; Ng, W.Y.Y.; Choi, R.C.Y.; Dong, T.T.X.; Wang, T.; Lau, D.T.W.; et al. The establishment of a highly sensitive method in detecting ketamine and norketamine simultaneously in human hairs by HPLC-Chip-MS/MS. Forensic Sci. Int. 2011, 208, 53-58. [CrossRef] [PubMed]

101. Harun, N.; Anderson, R.A.; Cormack, P.A.G. Analysis of ketamine and norketamine in hair samples using molecularly imprinted solid-phase extraction (MISPE) and liquid chromatography-tandem mass spectrometry (LC-MS/MS). Anal. Bioanal. Chem. 2010, 396, 2449-2459. [CrossRef] [PubMed]

102. Leong, H.S.; Tan, N.L.; Lui, C.P.; Lee, T.K. Evaluation of ketamine abuse using hair analysis: Concentration trends in a Singapore population. J. Anal. Toxicol. 2010, 29, 314-318. [CrossRef] [PubMed]

103. Tabernero, M.J.; Felli, M.L.; Bermejo, A.M.; Chiarotti, M. Determination of ketamine and amphetamines in hair by LC/MS/MS. Anal. Bioanal. Chem. 2009, 395, 2547-2557. [CrossRef]

104. Wu, Y.-H.; Lin, K.; Chen, S.-C.; Chang, Y.-Z. Integration of GC/EI-MS and GC/NCI-MS for simultaneous quantitative determination of opiates, amphetamines, MDMA, ketamine, and metabolites in human hair. J. Chromatogr. B 2008, 870, 192-202. [CrossRef] [PubMed]

105. Wu, Y.; Lin, K.; Chen, S.; Chang, Y. Simultaneous quantitative determination of amphetamines, ketamine, opiates and metabolites in human hair by gas chromatography/mass spectrometry. Rapid Commun. Mass Spectrom. 2008, 22, 887-897. [CrossRef]

106. Xiang, P.; Shen, M.; Zhuo, X. Hair analysis for ketamine and its metabolites. Forensic Sci. Int. 2006, 162, 131-134. [CrossRef] [PubMed]

107. Jang, M.; Kim, J.; Shin, I.; Kang, S.; Choi, H.; Yang, W. Simultaneous determination of methylphenidate and ritalinic acid in hair using LC-MS/MS. Forensic Sci. Int. 2019, 294, 183-188. [CrossRef] [PubMed]

108. Kłys, M.; Rojek, S.; Bolechała, F. Determination of oxcarbazepine and its metabolites in postmortem blood and hair by means of liquid chromatography with mass detection (HPLC/APCI/MS). J. Chromatogr. B 2005, 825, 38-46. [CrossRef]

109. Frison, G.; Favretto, D.; Tedeschi, L.; Ferrara, S.D. Detection of thiopental and pentobarbital in head and pubic hair in a case of drug-facilitated sexual assault. Forensic Sci. Int. 2003, 133, 171-174. [CrossRef]

110. Wishart, D.S.; Feunang, Y.D.; Guo, A.C.; Lo, E.J.; Marcu, A.; Grant, J.R.; Sajed, T.; Johnson, D.; Li, C.; Sayeeda, Z.; et al. DrugBank 5.0: A major update to the DrugBank database for 2018. Nucleic Acids Res. 2018, 46, D1074-D1082. [CrossRef]

111. Stowe, G.N.; Paulsen, R.B.; Hill, V.A.; Schaffer, M.I.; Corporation, P.; Way, U.; City, C. A retrospective analysis of selected opioids in hair of workplace drug testing subjects. J. Anal. Toxicol. 2019, 43, 553-563. [CrossRef]

112. Baselt, R.C. Disposition of Toxic Drugs and Chemicals in Man, 10th ed.; Biomedical Publications: Seal Beach, CA, USA, 2014; pp. 1846-1848.

113. Schulz, M.; Iwersen-Bergmann, S.; Andresen, H.; Schmoldt, A. Therapeutic and toxic blood concentrations of nearly 1000 drugs and other xenobiotics. Crit. Care 2012, 16, 2-5. [CrossRef] [PubMed]

114. Ramírez, M.; Baumgartner, W.A.; Wille, S.M.R.; Farabee, D.; Samyn, N.; Baumgartner, A.M.; Fernández, M.d.M.R.; Baumgartner, W.A.; Wille, S.M.R.; Farabee, D.; et al. A different insight in hair analysis: Simultaneous measurement of antipsychotic drugs and metabolites in the protein and melanin fraction of hair from criminal justice patients. Forensic Sci. Int. 2020, $312,110337$. [CrossRef]

115. Skopp, G.; Kniest, A.; Haisser, J.; Mann, K.; Hermann, D. Buprenorphine and norbuprenorphine findings in hair during constant maintenance dosage. Int. J. Legal Med. 2011, 125, 277-281. [CrossRef] [PubMed]

116. Baumgartner, W.A.; Hill, V.A.; Hillb, V.A. Sample preparation techniques. Forensic Sci. Int. 1993, 63, 121-135. [CrossRef] 